\title{
Quantum symmetries in orbifolds and decomposition
}

\author{
Daniel G. Robbins, ${ }^{a}$ Eric Sharpe $^{b}$ and Thomas Vandermeulen ${ }^{a}$ \\ ${ }^{a}$ Department of Physics, University at Albany, \\ Albany, NY 12222, U.S.A. \\ ${ }^{b}$ Department of Physics MC 0435, Virginia Tech, \\ 850 West Campus Drive, Blacksburg, VA 24061, U.S.A. \\ E-mail: dgrobbins@albany.edu, ersharpe@vt.edu, \\ tvandermeulen@albany .edu
}

ABSTRACT: In this paper, we introduce a new set of modular-invariant phase factors for orbifolds with trivially-acting subgroups, analogous to discrete torsion and generalizing quantum symmetries. After describing their basic properties, we generalize decomposition to include orbifolds with these new phase factors, making a precise proposal for how such orbifolds are equivalent to disjoint unions of other orbifolds without trivially-acting subgroups or one-form symmetries, which we check in numerous examples.

Keywords: Conformal Field Theory, Gauge Symmetry, Anomalies in Field and String Theories

ArXIV EPRINT: 2107.12386 


\section{Contents}

1 Introduction 1

2 Quantum symmetries of the $\Gamma$ orbifold 3

2.1 Review of ordinary quantum symmetries 3

2.2 Basics of general construction 4

2.3 Special case of discrete torsion $\quad 6$

2.4 General case 8

3 General conjecture for decomposition $\quad 13$

4 Examples $\quad \mathbf{1 5}$

$\begin{array}{lll}\text { 4.1 Extension of cyclic groups to larger cyclic groups } & 15\end{array}$

$\begin{array}{lll}\text { 4.1.1 } & \text { Extension of } \mathbb{Z}_{2} \text { to } \mathbb{Z}_{4} & 15\end{array}$

$\begin{array}{lll}\text { 4.1.2 } & \text { Extension of } \mathbb{Z}_{2} \text { by } \mathbb{Z}_{k} & 16\end{array}$

$\begin{array}{lll}\text { 4.1.3 } & \text { Extension of } \mathbb{Z}_{3} \text { to } \mathbb{Z}_{9} & 18\end{array}$

$\begin{array}{lll}4.2 & \text { Extension of } \mathbb{Z}_{2} \times \mathbb{Z}_{2} \text { to } \mathbb{Z}_{2} \times \mathbb{Z}_{4} & 19\end{array}$

$\begin{array}{lll}\text { 4.2.1 Without discrete torsion } & 19\end{array}$

4.2.2 With discrete torsion 21

$\begin{array}{lll}4.2 .3 & \text { Summary } & 22\end{array}$

4.3 Extension of $\mathbb{Z}_{2} \times \mathbb{Z}_{2}$ to $D_{4}$

4.3.1 Without discrete torsion 24

$\begin{array}{lll}4.3 .2 & \text { With discrete torsion } & 25\end{array}$

$\begin{array}{lll}4.3 .3 & \text { Summary } & 27\end{array}$

4.4 Extension of $\mathbb{Z}_{2} \times \mathbb{Z}_{4}$ to $\mathbb{Z}_{4} \rtimes \mathbb{Z}_{4}$

4.4.1 Without discrete torsion 28

4.4.2 With discrete torsion 30

$\begin{array}{lll}\text { 4.4.3 Summary } & 32\end{array}$

4.5 Extension of $D_{4}$ to $\mathbb{Z}_{4} \rtimes \mathbb{Z}_{4}$

4.5.1 Without discrete torsion 32

4.5.2 With discrete torsion 35

4.5.3 Summary 36

4.6 Extension of $\mathbb{Z}_{2} \times \mathbb{Z}_{2}$ to $D_{4} \times \mathbb{Z}_{2} \times \mathbb{Z}_{2}$

4.6.1 Without discrete torsion 37

4.6.2 With discrete torsion in $\mathbb{Z}_{2} \times \mathbb{Z}_{2} \subset \Gamma \quad 38$

4.6.3 With discrete torsion in $D_{4} \subset \Gamma \quad 39$

4.6.4 Summary 40

5 Conclusions $\quad 40$

A Notes on conventional quantum symmetries in orbifolds 41

B Triviality of $\pi^{*}\left(d_{2} B\right)$ in cohomology 42 
D Non-central extensions $\quad 45$

$\begin{array}{lll}\text { D.1 Basics } & 45\end{array}$

$\begin{array}{lll}\text { D.2 Example } & 47\end{array}$

\section{Introduction}

In an orbifold, it is an old story that one has the option of adding modular-invariant phases such as discrete torsion, which in a $\Gamma$ orbifold are classified by $H^{2}(\Gamma, \mathrm{U}(1))$. Recently, there has been renewed interest in orbifolds and their anomalies, see e.g. [1-9]. As outlined in e.g. $[1,4]$, and as will be explained in greater detail in our upcoming work [10], recent proposals for anomaly resolution in orbifolds $[2,4]$ implicitly require that the 'resolved' orbifolds use new modular-invariant degrees of freedom, analogous to (but distinct from) discrete torsion. It is the purpose of this paper to describe those novel modular-invariant degrees of freedom explicitly.

These new degrees of freedom generalize quantum symmetries of orbifolds [11, 12], for which reason we use the same nomenclature, and are specific to orbifolds in which a subgroup of the orbifold group acts trivially on the original space. Unlike ordinary quantum symmetries, the quantum symmetries we shall be focused on do not always arise from discrete torsion, and so define new modular-invariant phases - but in which the modular invariance is achieved in a novel fashion.

Specifically, these new degrees of freedom arise in the case that a subgroup $K$ of the orbifold group $\Gamma$ acts trivially. These additional modular invariant phases, which we call "quantum symmetries," are classified by $H^{1}\left(G, H^{1}(K, \mathrm{U}(1))\right)$, where $G=\Gamma / K$ acts effectively. It can happen that a subset of these phases may be equivalent to a subset of discrete torsion $H^{2}(\Gamma, \mathrm{U}(1))$, but in general these quantum symmetry phases are not the same as discrete torsion. For example, consider an orbifold by $\Gamma \cong \mathbb{Z}_{4}$ with a trivially acting $K \cong \mathbb{Z}_{2}$ subgroup, as is discussed in section 4.1.1. In this case, there is no discrete torsion ( since $H^{2}\left(\mathbb{Z}_{4}, \mathrm{U}(1)\right) \cong 1$ ), but there are two possible choices for quantum symmetry phases (since $\left.H^{1}\left(G, H^{1}(K, \mathrm{U}(1))\right)=\mathbb{Z}_{2}\right)$.

The purpose of this paper is both to explore these new degrees of freedom, which are of independent interest, and also to set the stage for their application to anomaly resolution, which will be explored in detail in [10]. We will offer a conjecture for the result of performing a gauging with a given choice of phases, at least in the case that $K$ is a central subgroup of $\Gamma$.

Before continuing, we should mention that there exist different generalizations of quantum symmetries in the literature. For example, [3] utilizes fusion categories to build a generalization with the property that for nonabelian orbifolds, orbifolding by the (fusion category) quantum symmetry returns the original space. Our generalization, by contrast, does not involve fusion categories (and when applied to nonabelian orbifolds need not return the original space), but will instead provide new degrees of freedom in orbifolds with trivially-acting subgroups. 
Orbifolds with trivially-acting subgroups, and more generally gauge theories with trivially-acting subgroups, such as abelian gauge theories with nonminimal charges, were explored in [13-15] as part of a program of developing string compactifications on certain generalized spaces known as stacks and gerbes. In modern language, a gerbe is a fiber bundle in which the fibers are 'groups' of one-form symmetries, hence a sigma model on a gerbe naturally admits a one-form symmetry corresponding to translation along the fibers.

One of the most important outcomes of that work was the discovery of decomposition, first described in [16], an equivalence between two-dimensional theories with one-form symmetries (and various generalizations) and disjoint unions of other two-dimensional theories. Decomposition has since been applied to a number of areas, including GromovWitten theory [17-22], gauged linear sigma models [23-29], mirror symmetry [16, 30-33] and heterotic string compactifications [34]. See e.g. [9, 35-39] for more recent work, on topics ranging from elliptic genera to higher-dimensional analogues.

Naturally, after introducing more general quantum symmetries in orbifolds with triviallyacting subgroups, in this paper we will also describe decomposition in such theories. This is both for some semblance of completeness, as well as because decomposition will play a crucial role in the application to anomalies, as we will discuss in [10].

We begin in section 2 by describing these new more general quantum symmetries and their basic properties, such as their relation to older notions of quantum symmetries, as well as the modular invariance of these new phases. In section 3 we then conjecture the form that decomposition takes in these theories, the modification required by the presence of a quantum symmetry.

In section 4 we explore a number of examples, both to demonstrate quantum symmetries explicitly, as well as to explore the effect of decomposition.

In a series of appendices, we collect some related information. In appendix A, we review conventional quantum symmetries in this framework, and how orbifolds-of-orbifolds returning the original theory (or a variant thereof) is a version of decomposition.

Appendix $\mathrm{B}$ is a technical demonstration that the pullback of the quantity $d_{2} B$ (which plays a role in our discussion) is trivial in cohomology. This will play an essential role in the application to anomalies discussed in [10], and also seems to be important for describing D-branes and open strings in these theories.

In appendix $\mathrm{C}$ we outline open strings and D-branes in orbifolds with quantum symmetries. To define a D-brane here requires a subtle generalization. For example, in an ordinary orbifold, to define a D-brane, one must give a group action on the D-brane, and if one adds discrete torsion, then that group action is projectivized. Here, quantum symmetries often require that associativity of the group action is (weakly) broken. In this appendix we outline basics.

In appendix $\mathrm{D}$ we outline some aspects of non-central extensions. In the rest of this paper, we assume that the full orbifold group $\Gamma$ is a central extension of the effectively-acting orbifold $G$, meaning that the trivially-acting subgroup lies within the center of $\Gamma$. At least much of the structure we describe appears to generalize to non-central extensions, and we outline some details here. 
Finally, a remark on nomenclature. Across our several papers on these matters, we have sometimes mixed additive and multiplicative notations. For example, a trivial quantum symmetry $B$ is sometimes written as $B=0$ and other times is written as $B(g)=1$ for all $g \in G$.

\section{Quantum symmetries of the $\Gamma$ orbifold}

\subsection{Review of ordinary quantum symmetries}

Let us begin with a short review of quantum symmetries in orbifolds. Given a $G$ orbifold, the quantum symmetry (see e.g. [11, section 8.5], [12]) multiplies twist fields by phases, so as to leave correlation functions invariant. If $G$ is abelian, the quantum symmetry $\hat{G} \cong G$; if $G$ is nonabelian, the quantum symmetry $\hat{G}$ is the abelianization $G /[G, G]$.

A famous consequence of quantum symmetries is that they can undo the orbifold: for $G$ abelian, orbifolding the original orbifold by the quantum symmetry returns the original theory. (For $G$ nonabelian, to recover the original theory one must work with fusion categories, see e.g. [3]; we shall take a different direction in this paper.)

Those two subsequent orbifolds, the orbifold of $[X / G]$ by the quantum symmetry $\hat{G}$, can equivalently be described as a single orbifold $[X /(G \times \hat{G})]$ with discrete torsion, which provides the phases produced by the quantum symmetry. The group $\hat{G}$ acts trivially on $X$; it only acts via $G$-twisted sector phases. For example, if $G=\mathbb{Z}_{n}$, then orbifolding by the quantum symmetry is equivalent to an orbifold by $\mathbb{Z}_{n} \times \mathbb{Z}_{n}$ with discrete torsion in $H^{2}\left(\mathbb{Z}_{n} \times \mathbb{Z}_{n}, \mathrm{U}(1)\right) \cong \mathbb{Z}_{n}$, where one $\mathbb{Z}_{n}$ factor acts trivially on $X$ (though its contributions to the path integral are weighted by phases). ${ }^{1}$ (See for example [11, section 8.5] for an explicit discussion for the case $n=2$.)

The fact that such an orbifold is equivalent to the original space is a special case of the decomposition for orbifolds with discrete torsion discussed in [9]. For example, the case $n=2$ is discussed in detail in [9, section 5.1], where it is checked that, as a consequence of decomposition,

$$
\operatorname{QFT}\left(\left[X / \mathbb{Z}_{2} \times \mathbb{Z}_{2}\right]_{\text {d.t. }}\right)=\operatorname{QFT}(X) .
$$

In appendix A, we review the analogous result for general orbifold groups $G$, demonstrating that for a quantum symmetry group $\hat{G}=G /[G, G]$, the orbifold $[X / \Gamma]$ for $\Gamma=G \times \hat{G}$ with appropriate discrete torsion is equivalent to $[X /[G, G]]$.

In this paper, we generalize quantum symmetries to orbifolds $[X / \Gamma]$, where $\Gamma$ is no longer a product but rather a central extension, and more importantly, to phases which need not be determined by discrete torsion. Despite the fact that the phases do not come from discrete torsion, we will find that they nevertheless are modular invariant, giving us new modular-invariant phases to add to orbifolds, at least orbifolds in which a subgroup of the orbifold group acts trivially. We will also find other subtleties in these new phases for example, appendix $\mathrm{C}$ discusses how D-branes in such orbifolds appear to be defined by non-associative analogues of equivariant structures.

\footnotetext{
${ }^{1}$ See for example [11, section 8.5] for an explicit discussion for the case $n=2$. Another perspective can be found in [40], where the quantum symmetry and the iterated gauging are discussed in the language of boundaries and interfaces of a 3D Dijkgraaf-Witten TQFT.
} 
In the remainder of this section we will discuss these new phases generalizing quantum symmetries, and discuss basic properties such as their modular invariance.

\subsection{Basics of general construction}

Next, we will consider more general quantum symmetries arising in an orbifold $\Gamma$ that is a central extension of an effectively-acting group $G$ by a trivially-acting group $K$. (In appendix D we outline some of the basics needed to generalize to non-central extensions, which otherwise are left for future work.)

As in the ordinary notion, the idea behind a quantum symmetry is that $K$ acts trivially on $X$, but nontrivially on $G$-twisted sectors. However, since in general $\Gamma$ can be a nontrivial extension, the $G$-twisted sectors are not well-defined, so that description is imprecise. A better description is that the set of $\Gamma$-twisted sectors is acted upon ${ }^{2}$ by $K$, with phases determined by $B \in H^{1}\left(G, H^{1}(K, \mathrm{U}(1))\right)$, as follows. Since $K$ is central, an element $B$ is equivalent to a map

$$
B: G \times K \longrightarrow \mathrm{U}(1),
$$

which we use to give relations of the form ${ }^{3}$

$$
g z \underset{h}{\square_{h}}=B(\pi(h), z)\left(g \square_{h}\right), \quad g \square_{h z}=B(\pi(g), z)^{-1}\left(g \square_{h}\right),
$$

for $z \in K$ and $g, h \in \Gamma$ a commuting pair. (We assume that $B(g, 1)=1$ for all $g \in G$, and $B(1, k)=1$ for all $k \in K$.) This is the precise meaning of the intuition that $K$ acts trivially on $X$, but nontrivially on $G$-twisted sectors. The resulting phases are classified by elements of

$$
H^{1}\left(G, H^{1}(K, \mathrm{U}(1))\right)
$$

In this paper, we will see in examples that with this choice, the resulting $\Gamma$ orbifold is well-defined, and typically has the form of (copies) of an orbifold by a subgroup of $G$. We will make a precise general prediction for the form of any such $\Gamma$ orbifold, which we will check extensively.

Before going on, let us check that the prescription above for quantum symmetries derived from an element of $H^{1}\left(G, H^{1}(K, \mathrm{U}(1))\right)$ is invariant under modular transformations. Begin with the relation

$$
g z \underset{h}{\square}=B(\pi(h), z)(g \underset{h}{\square}),
$$

and perform a modular transformation by

$$
\left[\begin{array}{ll}
a & b \\
c & d
\end{array}\right] \in \mathrm{SL}(2, \mathbb{Z})
$$

\footnotetext{
${ }^{2}$ Technically, the set of genus-one twisted sectors in the $\Gamma$ orbifold forms a torsor under $K$.

${ }^{3}$ Note that the relations are almost but not quite symmetric under an $\mathrm{S}$ transformation. This can be derived from modular transformations.
} 
Under this modular transformation

$$
\begin{gathered}
g z \underset{h}{\square} \mapsto(g z)^{a} h^{b} \underset{(g z)^{c} h^{d}}{\square}, \\
g \square_{h} \mapsto g^{a} h^{b} \underset{g^{c} h^{d}}{\square},
\end{gathered}
$$

and from the relation $(2.3)$,

$$
\begin{aligned}
(g z)^{a} h^{b} \underset{(g z)^{c} h^{d}}{\square} & =B\left(\pi\left(g^{c} h^{d}\right), z^{a}\right)\left(\begin{array}{ll}
g^{a} h^{b} & \square \\
& (g z)^{c} h^{d}
\end{array}\right), \\
& =\frac{B\left(\pi\left(g^{c} h^{d}\right), z^{a}\right)}{B\left(\pi\left(g^{a} h^{b}\right), z^{c}\right)}\left(g^{a} h^{b} \underset{g^{c} h^{d}}{\square}\right) .
\end{aligned}
$$

Since $B \in H^{1}\left(G, H^{1}(K, \mathrm{U}(1))\right)$, and $K$ is assumed central, we know that

$$
\begin{aligned}
& B\left(g, z_{1} z_{2}\right)=B\left(g, z_{1}\right) B\left(g, z_{2}\right), \\
& B\left(g_{1} g_{2}, z\right)=B\left(g_{1}, z\right) B\left(g_{2}, z\right),
\end{aligned}
$$

hence

$$
\begin{aligned}
\frac{B\left(\pi\left(g^{c} h^{d}\right), z^{a}\right)}{B\left(\pi\left(g^{a} h^{b}\right), z^{c}\right)} & =\frac{B\left(\pi\left(g^{c} h^{d}\right), z\right)^{a}}{B\left(\pi\left(g^{a} h^{b}, z\right)^{c}\right.}, \\
& =\frac{B\left(\pi\left(g^{c a} h^{d a}\right), z\right)}{B\left(\pi\left(g^{a c} h^{b c}\right), z\right)}, \\
& =B\left(\pi\left(h^{a d-b c}\right), z\right)=B(\pi(h), z) .
\end{aligned}
$$

Thus, we see that

$$
(g z)^{a} h^{b} \underset{(g z)^{c} h^{d}}{\square}=B(\pi(h), z)\left(g^{a} h^{b} \underset{g^{c} h^{d}}{\square}\right),
$$

precisely consistent with a modular transformation of the identity (2.5).

In section 2.1 we reviewed ordinary quantum symmetries, described by trivial extensions $\Gamma=G \times K$, and which were produced by discrete torsion. The relationship can be made precise using the exact sequence

$$
\left(\operatorname{Ker} i^{*} \subset H^{2}(\Gamma, \mathrm{U}(1))\right) \stackrel{\beta}{\longrightarrow} H^{1}\left(G, H^{1}(K, \mathrm{U}(1))\right) \stackrel{d_{2}}{\longrightarrow} H^{3}(G, \mathrm{U}(1)),
$$

where $\iota: K \rightarrow \Gamma$ is inclusion and in the sequence above,

$$
\iota^{*}: H^{2}(\Gamma, \mathrm{U}(1)) \longrightarrow H^{2}(K, \mathrm{U}(1)) .
$$

(This is part of a seven-term exact sequence [41] slightly extending the inflation-restriction sequence (see e.g. [42], [43, example 6.8.3], [44, section I.6], [45, section 3.3]), that is discussed in greater detail in our previous work [9].) For later use, the maps $\beta$ and $d_{2}$ are given as

$$
\beta(\omega)(\pi(g), z)=\frac{\omega(g, z)}{\omega(z, g)},
$$


(for $g \in \Gamma, z \in K$, with $\omega$ a cocycle representing an element of $H^{2}(\Gamma, \mathrm{U}(1)$ ) corresponding to discrete torsion) as we shall discuss in detail in the next section, as well as [9, appendix C], and

$$
\left(d_{2} B\right)\left(\bar{g}_{1}, \bar{g}_{2}, \bar{g}_{3}\right)=B\left(\bar{g}_{1}, s_{2} s_{3} s_{23}^{-1}\right),
$$

where $\bar{g}_{i} \in G, s_{i}=s\left(\bar{g}_{i}\right)$ for $s: G \rightarrow \Gamma$ a section, as discussed in [41, section 8]. (The quantity $s_{2} s_{3} s_{23}^{-1}$ is the extension class in $H^{2}(G, K)$ of $\Gamma$, and so vanishes in cohomology if the extension splits.)

In any event, a quantum symmetry $B \in H^{1}\left(G, H^{1}(K, \mathrm{U}(1))\right)$ arises from discrete torsion precisely when it is in the image of $\beta$ in the sequence (2.17) above. Furthermore, the reader should note from the sequence (2.17) that not every quantum symmetry $B$ can be so described - those for which $d_{2} B$ is nontrivial, are not in the image of $\beta$ and so are not given by discrete torsion.

\subsection{Special case of discrete torsion}

An important set of special cases are those in which the quantum symmetry, defined by $B \in H^{1}\left(G, H^{1}(K, \mathrm{U}(1))\right)$, arises from discrete torsion $\omega \in H^{2}(\Gamma, \mathrm{U}(1))$, meaning that $B$ is in the image of $\beta$ in the sequence (2.17). In this section we will verify that mathematical result directly in physics. (See also [9, appendix C] for another discussion of $\beta$.)

We have described quantum symmetries as elements $B \in H^{1}\left(G, H^{1}(K, \mathrm{U}(1))\right.$ that relate genus-one twisted sectors as

$$
g z \underset{h}{\square}=B(\pi(h), z)(g \underset{h}{\square}),
$$

for $g, h$ commuting elements of $\Gamma$, and $z \in K$. If $K$ acts trivially on the original theory (albeit not on $G$-twisted sectors), then this implies a relation between phases derived from discrete torsion $\omega \in H^{2}(\Gamma, \mathrm{U}(1))$, specifically

$$
\frac{\omega(g z, h)}{\omega(h, g z)}=B(\pi(h), z) \frac{\omega(g, h)}{\omega(h, g)},
$$

or equivalently,

$$
B(\pi(h), z)=\frac{\omega(g z, h)}{\omega(h, g z)} \frac{\omega(h, g)}{\omega(g, h)},
$$

where we have used the fact that discrete torsion assigns the phase

$$
\epsilon_{\mathrm{dt}}(g, h)=\frac{\omega(g, h)}{\omega(h, g)}
$$

to the genus-one diagram

$$
g \square_{h}
$$

defined by commuting $g, h$. In deriving this expression, we have merely assumed that the twisted sectors are well-defined, meaning that $h$ commutes with both $g$ and $g z$.

In order to be consistent, the right-hand-side of equation (2.23) must not depend on $g$, and can only depend upon $\pi(h)$ instead of all of $h$. We will see that the first statement 
is always true, and the second is true so long as the restriction of $\omega$ to $H^{2}(K, \mathrm{U}(1))$ is trivial. (If the restriction is not trivial, then $B=B(h, z)$ and so defines an element of $H^{1}\left(\Gamma, H^{1}(K, \mathrm{U}(1))\right)$ instead of $H^{1}\left(G, H^{1}(K, \mathrm{U}(1))\right)$.)

Both of these well-definedness statements are a consequence of the fact that, as noted in e.g. [46, equ'n (42)], for the genus-one discrete torsion phases

$$
\epsilon_{\mathrm{dt}}(g, h)=\frac{\omega(g, h)}{\omega(h, g)}
$$

(for $g, h$ commuting) one has

$$
\epsilon_{\mathrm{dt}}(x, y) \epsilon_{\mathrm{dt}}(x, z)=\epsilon_{\mathrm{dt}}(x, y z) .
$$

To demonstrate that $B(\pi(h), z)$ is independent of $g$, one rearranges

$$
\epsilon_{\mathrm{dt}}(h, g) \epsilon_{\mathrm{dt}}(h, z)=\epsilon_{\mathrm{dt}}(h, g z),
$$

to quickly find that

$$
\frac{\omega(g z, h)}{\omega(h, g z)} \frac{\omega(h, g)}{\omega(g, h)}=\frac{\omega(z, h)}{\omega(h, z)} .
$$

The left-hand side is equation (2.23) for $B$, and the right-hand side is the restriction of $B$ to $g=1$. Since they are equal, we see that, as advertised, $B$ is independent of $g$, and in particular, we can write

$$
B(\pi(h), z)=\frac{\omega(z, h)}{\omega(h, z)}=\epsilon_{\mathrm{dt}}(z, h) .
$$

In a similar fashion, one can show that so long as the restriction of $\omega$ to $H^{2}(K, \mathrm{U}(1))$ is trivial, $B$ only depends on $\pi(h)$, not $h$. Specifically, suppose that $h^{\prime}=h k$ for $k \in K$. Then, using the identity (2.27), we have that

$$
\epsilon_{\mathrm{dt}}\left(z, h^{\prime}\right)=\epsilon_{\mathrm{dt}}(z, h k)=\epsilon_{\mathrm{dt}}(z, h) \epsilon_{\mathrm{dt}}(z, k),
$$

but since $z, k$ are both in $K$, so long as the restriction of $\omega$ to $H^{2}(K, \mathrm{U}(1))$ is trivial, $\epsilon(z, k)=1$, hence

$$
\epsilon_{\mathrm{dt}}\left(z, h^{\prime}\right)=\epsilon_{\mathrm{dt}}(z, h),
$$

and so $B$ depends only upon $\pi(h)$, not $h$.

We can also make contact with another expression for a phase derived from discrete torsion. As the expression (2.23) for $B$ is independent of $g$, without loss of generality we can take $g=h^{-1}$, then from (2.23), we find another expression for $B$ :

$$
B(\pi(h), z)=\frac{\omega\left(h^{-1} z, h\right)}{\omega\left(h, h^{-1} z\right)} \frac{\omega\left(h, h^{-1}\right)}{\omega\left(h^{-1}, h\right)} .
$$

Now,

$$
\frac{\omega\left(h, h^{-1}\right)}{\omega\left(h^{-1}, h\right)}=1
$$

and

$$
\beta(\pi(h), \omega)(z)=\frac{\omega\left(h, h^{-1} z\right)}{\omega\left(h^{-1} z, h\right)}
$$


was the phase factor defined by discrete torsion $\omega$ in $[9$, appendix $\mathrm{C}]$, so we see that

$$
B(\pi(h), z)=\frac{1}{\beta(\pi(h), \omega)(z)} .
$$

In [9] this phase factor was computed from the mathematics of the Lyndon-Hochschild-Serre spectral sequence, whereas here we have seen a simple physical realization.

Thus, we see that so long as the restriction of discrete torsion to $H^{2}(K, \mathrm{U}(1))$ is trivial, discrete torsion defines a quantum symmetry $B \in H^{1}\left(G, H^{1}(K, \mathrm{U}(1))\right.$, given by $B=1 / \beta(\omega)$. that $K$ is central in $\Gamma$.

Conversely, if $d_{2} B=1$, then we can find a corresponding element of discrete torsion, as follows. In this case, there exists a two-cochain $\lambda$ such that

$$
B\left(g_{1}, s_{2} s_{3} s_{23}^{-1}\right)=\frac{\lambda\left(g_{2}, g_{3}\right)}{\lambda\left(g_{1} g_{2}, g_{3}\right)} \frac{\lambda\left(g_{1}, g_{2} g_{3}\right)}{\lambda\left(g_{1}, g_{2}\right)} .
$$

Then, define $\omega$ by

$$
\omega\left(s_{1} k_{1}, s_{2} k_{2}\right)=\frac{\lambda\left(g_{1}, g_{2}\right)}{B\left(g_{1}, k_{2}\right)} .
$$

Now. only some elements of $H^{1}\left(G, H^{1}(K, \mathrm{U}(1))\right)$ can be written as $1 / \beta(\omega)$ for some $\omega$ in $H^{2}(\Gamma, \mathrm{U}(1))$. In particular, in this paper we are interested in $B$ in $H^{1}\left(G, H^{1}(K, \mathrm{U}(1))\right)$ whose image in $H^{3}(G, \mathrm{U}(1))$ is nontrivial, and those $B$ are not in the image of $\omega$, as follows from the exact sequence (2.17), which was reviewed in greater detail in [9].

For reference elsewhere, from the sequence (2.17), for $B \in H^{1}\left(G, H^{1}(K, \mathrm{U}(1))\right)$ such that $d_{2} B \neq 1$ (and so are not determined by discrete torsion), although $d_{2} B$ is a nontrivial element of $H^{3}(G, \mathrm{U}(1))$, we will show in appendix B that $\pi^{*}\left(d_{2} B\right)$ is trivial in $H^{3}(\Gamma, \mathrm{U}(1))$ - at least, trivial in cohomology, though not necessarily identically 1 .

In passing, note that for $B$ determined by discrete torsion $\omega \in H^{2}(\Gamma, \mathrm{U}(1))$, as $\epsilon_{\mathrm{dt}}(g, h)=$ $\epsilon_{\mathrm{dt}}(h, g)^{-1}$, to be consistent, relation (2.21) also requires

$$
h \square_{g z}=B(\pi(h), z)^{-1}(h \underset{g}{\square}),
$$

exactly as we saw is required for modular invariance in section 2.2.

\subsection{General case}

As noted above, not all $B \in H^{1}\left(G, H^{1}(K, \mathrm{U}(1))\right)$ are determined by discrete torsion in $H^{2}(\Gamma, \mathrm{U}(1))$. However, we would like an analogous description, in order to e.g. make sense of D-branes in these orbifolds. In this section we will discuss how one can compute cochains $\omega$ (not cocycles) whose corresponding phases

$$
\epsilon(g, h)=\frac{\omega(g, h)}{\omega(h, g)}
$$

encode $B$. 
To that end, first pick a section $s: G \rightarrow \Gamma$, and note that every $g \in \Gamma$ can be uniquely written in the form $g=s(\pi(g)) k_{g}$, where $k_{g}$ is in (the image of) $K$, and $\pi: \Gamma \rightarrow G$. Then, as previously discussed, any given twisted sector

$$
g \square_{h}=s(\pi(g)) k_{g} \underset{s(\pi(h)) k_{h}}{\square}
$$

is proportional to a sector without the factors of $k$ :

$$
s(\pi(g)) k_{g} \underset{s(\pi(h)) k_{h}}{\square}=\frac{B\left(\pi(h), k_{g}\right)}{B\left(\pi(g), k_{h}\right)}\left(\begin{array}{lll}
s(\pi(g)) & \square \\
& & \square(\pi(h))
\end{array}\right) .
$$

Since, aside from the quantum symmetry, $K$ otherwise acts trivially, we could equivalently say that we multiply the

$$
g \underset{h}{\square}
$$

by a phase

$$
\epsilon(g, h) \equiv \frac{B\left(\pi(h), s(\pi(g))^{-1} g\right)}{B\left(\pi(g), s(\pi(h))^{-1} h\right)}
$$

(We suspect, but have not carefully checked, that this is equivalent to including the coupling [4, equ'n (2.31)].)

As a consistency check, it is straightforward to show that, for example,

$$
\frac{g z \square h}{g \square h}=\frac{\epsilon(g z, h)}{\epsilon(g, h)}=B(\pi(h), z)
$$

using the fact that $B$ is a homomorphism (2.11), (2.12). Note that although a particular realization in terms of phases $\epsilon$ depends upon the choice of section $s$, the ratio of phase factors is independent of the choice of $s$.

The idea of multiplying a given sector by a phase factor is clearly analogous to that in discrete torsion, and obeys similar constraints. For example, it is also straightforward, using the fact that $B$ is a homomorphism (2.11), to demonstrate that

1. $\epsilon(g, g)=1$,

2. $\epsilon(g, h)=\epsilon(h, g)^{-1}$,

3. $\epsilon\left(g, h_{1} h_{2}\right)=\epsilon\left(g, h_{1}\right) \epsilon\left(g, h_{2}\right)\left(\left(d_{2} B\right)\left(\pi(g), \pi\left(h_{1}\right), \pi\left(h_{2}\right)\right)^{-1}\right.$,

for the phases $\epsilon$ defined in (2.44), and where [41, section 8]

$$
\left(d_{2} B\right)\left(\bar{g}_{1}, \bar{g}_{2}, \bar{g}_{3}\right)=B\left(\bar{g}_{1}, s\left(\bar{g}_{2}\right) s\left(\bar{g}_{3}\right) s\left(\bar{g}_{2} \bar{g}_{3}\right)^{-1}\right)
$$

for $\bar{g}_{1,2,3} \in G$, and where $d_{2}$ is the same map that appeared in the short exact sequence (2.17). In the special case that $d_{2} B=1$, these are the conditions that were previously derived on phases in [46, equ'ns (42)-(44)] to guarantee modular invariance, in the sense that

$$
\epsilon\left(g^{a} h^{b}, g^{c} h^{d}\right)=\epsilon(g, h)
$$


for

$$
\left[\begin{array}{ll}
a & b \\
c & d
\end{array}\right] \in \mathrm{SL}(2, \mathbb{Z}) .
$$

In more general cases, $d_{2} B$ acts as an obstruction to this identity.

Now, despite this obstruction, this theory can nevertheless still be modular invariant. To see this, the reader should note that the relation between the identity (2.47) and modular invariance is slightly subtle when $K$ acts trivially. In such a case, different sectors from possibly different $\mathrm{SL}(2, \mathbb{Z})$ orbits can contribute to the same effective orbifold sector. In particular, one can show that the partition function is modular invariant for any $B$, although for $d_{2} B \neq 1$ the phases $\epsilon$ themselves are not $\mathrm{SL}(2, \mathbb{Z})$-invariant.

We can see this as follows. For $\bar{g}=\pi(g), \bar{h}=\pi(h)$ the images in $G$ of a commuting pair of elements $g, h \in \Gamma$, define

$$
\bar{\epsilon}(\bar{g}, \bar{h})=\sum_{k_{1}, k_{2} \in K} \epsilon\left(s(\bar{g}) k_{1}, s(\bar{h}) k_{2}\right),
$$

where we assume that $K$ is central in $\Gamma$. In particular, $\bar{\epsilon}$ is the sum of the contributions to an effective- $G$-orbifold genus one sector defined by the commuting pair $\bar{g}, \bar{h}$. Then, we claim that for any

$$
\left[\begin{array}{ll}
a & b \\
c & d
\end{array}\right] \in \mathrm{SL}(2, \mathbb{Z})
$$

we have

$$
\bar{\epsilon}\left(\bar{g}^{a} \bar{h}^{b}, \bar{g}^{c} \bar{h}^{d}\right)=\bar{\epsilon}(\bar{g}, \bar{h}) .
$$

To see this, first note that

$$
\begin{aligned}
\bar{\epsilon}(\bar{g}, \bar{h}) & =\sum_{k_{1}, k_{2}} \epsilon\left(s(\bar{g}) k_{1}, s(\bar{h}) k_{2}\right), \\
& =\sum_{k_{1}, k_{2}} \frac{B\left(\bar{h}, k_{1}\right)}{B\left(\bar{g}, k_{2}\right)}
\end{aligned}
$$

then we compute

$$
\begin{aligned}
\bar{\epsilon}\left(\bar{g}^{a} \bar{h}^{b}, \bar{g}^{c} \bar{h}^{d}\right) & =\sum_{k_{1}, k_{2}} \frac{B\left(\bar{g}^{c} \bar{h}^{d}, k_{1}\right)}{B\left(\bar{g}^{a} \bar{h}^{b}, k_{2}\right)}, \\
& =\sum_{k_{1}, k_{2}} \frac{B\left(\bar{g}, k_{1}\right)^{c} B\left(\bar{h}, k_{1}\right)^{d}}{B\left(\bar{g}, k_{2}\right)^{a} B\left(\bar{h}, k_{2}\right)^{b}}, \\
& =\sum_{k_{1}, k_{2}} \frac{B\left(\bar{h}, k_{1}^{d} k_{2}^{-b}\right)}{B\left(\bar{g}, k_{2}^{a} k_{2}^{-c}\right)} .
\end{aligned}
$$

Finally, note that we can perform a change of basis, defining

$$
\ell_{1}=k_{1}^{d} k_{2}^{-b}, \quad \ell_{2}=k_{2}^{a} k_{1}^{-c},
$$


which can be inverted as

$$
k_{1}=\ell_{1}^{a} \ell_{2}^{b}, \quad k_{2}=\ell_{1}^{a} \ell_{2}^{d} .
$$

In the new basis, we can write

$$
\begin{aligned}
\bar{\epsilon}\left(\bar{g}^{a} \bar{h}^{b}, \bar{g}^{c} \bar{h}^{d}\right) & =\sum_{\ell_{1}, \ell_{2}} \frac{B\left(\bar{h}, \ell_{1}\right)}{B\left(\bar{g}, \ell_{2}\right)}, \\
& =\bar{\epsilon}(\bar{g}, \bar{h}),
\end{aligned}
$$

demonstrating that the theory defined by any $B$, not just those with $d_{2} B=1$, is modular invariant.

Thus, theories with quantum symmetries such that $d_{2} B \neq 1$ are modular-invariant, but the reasons are subtle. Modular invariance acts on both the $G$ twisted sectors as well as the $\Gamma$-twisted sectors. However, as

$$
\left(\pi\left(g^{a} h^{b}\right), \pi\left(g^{c} h^{d}\right)\right)=\left(\pi(g)^{a} \pi(h)^{b}, \pi(g)^{c}, \pi(h)^{d}\right),
$$

a modular transformation in $\Gamma$ will never slip between modular orbits in $G$; at most, it will exchange fibers over the same modular orbit of $G$. The effect of the quantum symmetry is to modify the coefficients of the $G$-twisted sector contributions, and although $\Gamma$-modularinvariance is broken if $d_{2} B \neq 1, G$-modular-invariance is not, as the effect of summing up the contributions from the action of $K$ can be arranged consistently in modular orbits of $G$.

To summarize, so far we have found that for these orbifolds with quantum symmetries, the phases (2.44) define modular-invariant phases, and so we see that, at least in orbifolds of this form, with a trivially-acting subgroup, discrete torsion is not the only possible modular-invariant phase one can add. (See also [49] for a discussion of momentum/winding lattice phases in orbifolds of non-simply-connected spaces, as another analogous example.)

As another consistency check, in the special case that $B$ is determined by discrete torsion $\omega$, that $B=1 / \beta(\omega)$, from (2.42), one expects

$$
\epsilon(g, h)=\frac{\epsilon_{\mathrm{dt}}(g, h)}{\epsilon_{\mathrm{dt}}(s(\pi(g)), s(\pi(h)))},
$$

where $\epsilon_{\mathrm{dt}}$ denotes the ratio of group 2-cocycles $\omega$ defining the genus-one phase arising in discrete torsion. Using multiplicative properties of $\epsilon_{\mathrm{dt}}$, as well as the assumption that its restriction to $K$ is trivial, it is straightforward to compute that

$$
\begin{aligned}
\epsilon(g, h) & =\frac{B\left(\pi(h), s(\pi(g))^{-1} g\right)}{B\left(\pi(g), s(\pi(h))^{-1} h\right)}=\frac{\epsilon_{\mathrm{dt}}\left(s(\pi(g))^{-1} g, h\right)}{\epsilon_{\mathrm{dt}}\left(s(\pi(h))^{-1} h, g\right)}, \\
& =\epsilon_{\mathrm{dt}}\left(s(\pi(g))^{-1}, h\right) \epsilon_{\mathrm{dt}}(g, h) \epsilon_{\mathrm{dt}}\left(g, s(\pi(h))^{-1} h\right) \\
& =\epsilon_{\mathrm{dt}}(g, h) \epsilon_{\mathrm{dt}}\left(s(\pi(g))^{-1}, h\right) \epsilon_{\mathrm{dt}}\left(s(\pi(g)), s(\pi(h))^{-1} h\right), \\
& =\epsilon_{\mathrm{dt}}(g, h) \frac{\epsilon_{\mathrm{dt}}\left(s(\pi(g)), s(\pi(h))^{-1} h\right)}{\epsilon_{\mathrm{dt}}(s(\pi(g)), h)} \\
& =\frac{\epsilon_{\mathrm{dt}}(g, h)}{\epsilon_{\mathrm{dt}}(s(\pi(g)), s(\pi(h)))},
\end{aligned}
$$

as expected. 
So far, given a general quantum symmetry, we have derived genus-one phase factors $\epsilon(g, h)$ that encode the quantum symmetry on twisted sectors. To make sense of D-branes, we need a bit more. Specifically, we need an analogue of group cocycles. Now, in general, these phase factors cannot be described by group cocycles, as that description only reproduces those quantum symmetries arising from discrete torsion. However, we can certainly construct group cochains representing the phases $\epsilon(g, h)$.

Specifically, recall a group cochain $\omega$ is a map $\Gamma \times \Gamma \rightarrow \mathrm{U}(1)$, not necessarily obeying a group cocycle condition. We can construct a set of group cochains $\omega(g, h)$ such that

$$
\epsilon(g, h)=\frac{\omega(g, h)}{\omega(h, g)}
$$

as follows. First, pick an ordering on the elements of the group $\Gamma$. (Different orderings will result in different cochains, but the same phase $\epsilon$.) Then, define

$$
\omega(g, h)=\left\{\begin{array}{cl}
\epsilon(g, h) & g \leq h, \\
1 & \text { else }
\end{array}\right.
$$

This construction is certainly not unique, but does demonstrate the existence of cochains $\omega$ that reproduce the phases $\epsilon$.

Another, potentially more useful, description of the cochain is as follows. Pick a section $s: G \rightarrow \Gamma$, with respect to which any $g \in \Gamma$ can be uniquely written $g=k s(\pi(g))$, for $k \in K$, then define

$$
\omega\left(k_{1} s\left(\pi\left(g_{1}\right)\right), k_{2} s\left(\pi\left(g_{2}\right)\right)\right) \equiv \frac{1}{B\left(\pi\left(g_{1}\right), k_{2}\right)} .
$$

Then,

$$
\begin{aligned}
\epsilon(z, h) & =\frac{\omega(z, h)}{\omega(h, z)}=\frac{B(\pi(h), z)}{B\left(1, s(\pi(h))^{-1} h\right)}, \\
& =B(\pi(h), z)
\end{aligned}
$$

using the fact that $B\left(1, s(\pi(h))^{-1} h\right)=1$.

Let us check when the cochain $\omega$ defined above is a cocycle. Let $g_{1,2,3}$ be three elements of $\gamma$, each written as $g_{i}=k_{i} s\left(\pi\left(g_{i}\right)\right)$. Then,

$$
\begin{aligned}
(d \omega)\left(g_{1}, g_{2}, g_{3}\right) & =\frac{\omega\left(g_{2}, g_{3}\right) \omega\left(g_{1}, g_{2} g_{3}\right)}{\omega\left(g_{1} g_{2}, g_{3}\right) \omega\left(g_{1}, g_{2}\right)}, \\
& =\frac{B\left(\pi\left(g_{1} g_{2}\right), k_{3}\right) B\left(\pi\left(g_{1}\right), k_{2}\right)}{B\left(\pi\left(g_{2}\right), k_{3}\right) B\left(\pi\left(g_{1}\right), k_{2} k_{3} s\left(\pi\left(g_{2}\right)\right) s\left(\pi\left(g_{3}\right)\right) s\left(\pi\left(g_{2} g_{3}\right)\right)^{-1}\right)}, \\
& =B\left(\pi\left(g_{1}\right), s\left(\pi\left(g_{2}\right)\right) s\left(\pi\left(g_{3}\right)\right) s\left(\pi\left(g_{2} g_{3}\right)\right)^{-1}\right)^{-1} .
\end{aligned}
$$

Now, from [41, section 8],

$$
\pi^{*}\left(d_{2} B\right)\left(g_{1}, g_{2}, g_{3}\right)=B\left(\pi\left(g_{1}\right), s\left(\pi\left(g_{2}\right)\right) s\left(\pi\left(g_{3}\right)\right) s\left(\pi\left(g_{2} g_{3}\right)\right)^{-1}\right),
$$

so we see that the cochain $\omega$ defined in (2.70) is a cocycle if and only if the pullback of $d_{2} B$ is trivial (not just in cohomology), and hence $B$ is in the image of $\beta$, using the exact sequence (2.17). 
In passing, it is straightforward to check that when $K$ is central, $\pi^{*}\left(d_{2} B\right)$ is a group 3-cocycle:

$$
\begin{aligned}
\left(d\left(\pi^{*} d_{2} B\right)\right)\left(g_{1}, g_{2}, g_{3}, g_{4}\right) & =\frac{\left(\pi^{*} d_{2} B\right)\left(g_{2}, g_{3}, g_{4}\right)}{\left(\pi^{*} d_{2} B\right)\left(g_{1} g_{2}, g_{3}, g_{4}\right)} \frac{\left(\pi^{*} d_{2} B\right)\left(g_{1}, g_{2} g_{3}, g_{4}\right)}{\left(\pi^{*} d_{2} B\right)\left(g_{1}, g_{2}, g_{3} g_{4}\right)}\left(\pi^{*} d_{2} B\right)\left(g_{1}, g_{2}, g_{3}\right), \\
& =\frac{B\left(\pi\left(g_{2}\right), s_{3} s_{4} s_{34}^{-1}\right)}{B\left(\pi\left(g_{1} g_{2}\right), s_{3} s_{4} s_{34}^{-1}\right)} \frac{B\left(\pi\left(g_{1}\right) s_{23} s_{4} s_{234}^{-1}\right)}{B\left(\pi\left(g_{1}\right), s_{2} s_{34} s_{234}^{-1}\right)} B\left(\pi\left(g_{1}\right), s_{2} s_{3} s_{23}^{-1}\right), \\
& =B\left(\pi\left(g_{1}\right), 1\right)=1,
\end{aligned}
$$

where for example $s_{a}=s\left(\pi\left(g_{a}\right)\right)$.

Furthermore, from equation (2.75) above, we see that $\pi^{*} d_{2} B$ is always trivial in cohomology in $H^{3}(\Gamma, \mathrm{U}(1))$, even if $d_{2} B$ is not trivial in $H^{3}(G, \mathrm{U}(1))$, at least for $K$ central. In appendix $\mathrm{B}$ we will demonstrate that even for non-central extensions, the pullback $\pi^{*} d_{2} B$ is trivial in cohomology.

\section{General conjecture for decomposition}

In this section we will relate orbifolds with quantum symmetries to simpler orbifolds, via a generalization of decomposition $[9,16,35,36]$. (Related observations concerning module categories have also been made in the mathematics literature on fusion categories, see e.g. [47, examples 7.4.10, 9.7.2].)

Previously, in $[9,16]$, it was possible to give a derivation of the form of decomposition by studying the structure of D-branes. In [16], this meant studying group actions on honest representations, and in [9], this meant studying group actions on projective representations. Unfortunately, we do not yet have a complete understanding of D-branes in orbifolds with quantum symmetries - see instead appendix C for partial results. As a result, we cannot give a derivation of the same form. Nevertheless, previous results do strongly constrain possible answers, so we are able to give a fairly full accounting of possibilities, which we check in numerous examples later in this paper. In our upcoming work [10] we will apply these ideas to resolutions of anomalies.

Let $\Gamma$ be a central extension of $G$ by (abelian) $K$, and suppose one has a quantum symmetry defined by $B \in H^{1}\left(G, H^{1}(K, \mathrm{U}(1))\right)$. We begin with the case that the orbifold can be described solely in terms of a quantum symmetry $B$. (Many choices of discrete torsion can be folded into $B$, as we have discussed, but not all - we will discuss more general cases shortly.) This case is tightly constrained by our previous results [9]. Specifically, there we discussed discrete torsion $\omega$ whose restriction to $K$ was trivial. We argued there that decomposition in the $\Gamma$ orbifold with discrete torsion was of the form

$$
\operatorname{QFT}\left([X / \Gamma]_{\omega}\right)=\operatorname{QFT}\left(\left[\frac{X \times \operatorname{Coker} \beta(\omega)}{\operatorname{Ker} \beta(\omega)}\right]_{\hat{\omega}}\right)
$$

for suitable choices of discrete torsion described in [9]. We can identify $\beta(\omega)^{-1}$ with a choice of quantum symmetry, and although not every quantum symmetry can be so described, this result does provide a large set of special cases. 
In order to reproduce that result, we conjecture in the present case that the $\Gamma$ orbifold, which we denote $[X / \Gamma]_{B}$, decomposition takes the form

$$
\operatorname{QFT}\left([X / \Gamma]_{B}\right)=\operatorname{QFT}\left(\left[\frac{X \times \widehat{\operatorname{Coker} B}}{\operatorname{Ker} B}\right]_{\hat{\omega}}\right),
$$

with discrete torsion $\hat{\omega}$ determined just as in decomposition $[9,16,35,36]$, meaning that for any irreducible representation $\rho$ of Coker $B$, we take $\hat{\omega}(\rho)$ to be the restriction to Ker $B$ of the image of the extension class of $\Gamma$, an element of $H^{2}(G, K)$, in $H^{2}(G, \mathrm{U}(1)$ ). (We are assuming, as elsewhere, that $K$ is central.)

As a special case, when $B$ is trivial, Ker $B=G$ and Coker $B=K$, so the prediction reduces to

$$
\operatorname{QFT}([X / \Gamma])=\operatorname{QFT}\left(\left[\frac{X \times \hat{K}}{G}\right]_{\hat{\omega}}\right),
$$

which matches the prediction of decomposition $[9,16,35,36]$ in this case.

We will explicitly verify the conjecture above in examples, by comparing the predictions to genus-one partition functions, in sections 4.1, 4.2.1, 4.3.1, 4.4.1, 4.5.1, 4.6.1.

Next, let us generalize this story slightly, by explicitly allowing for discrete torsion $\omega \in H^{2}(\Gamma, \mathrm{U}(1))$ in the $\Gamma$ orbifold, whose restriction to $K$ we will assume trivial, in addition to the quantum symmetry $B$. Following the pattern of [9], and mindful of special cases in which $B=1 / \beta\left(\omega^{\prime}\right)$ for some $\omega^{\prime}$, we break the analysis up into cases using the maps $\iota, \pi$ in

$$
1 \longrightarrow K \stackrel{\iota}{\longrightarrow} \Gamma \stackrel{\pi}{\longrightarrow} G \longrightarrow 1
$$

as follows:

1. Suppose that $\iota^{*} \omega \neq 0$ as an element of $H^{2}(K, \mathrm{U}(1))$. We do not have a conjecture for this case, though we will compute an example in section 4.6.2.

2. Suppose that $\iota^{*} \omega=0$ and $\beta(\omega) \neq 0$, where $\beta(\omega) \in H^{1}\left(G, H^{1}(K, \mathrm{U}(1))\right)$. Then,

$$
\operatorname{QFT}\left([X / \Gamma]_{B, \omega}\right)=\operatorname{QFT}\left(\left[\frac{X \times \operatorname{Coker}(B / \beta(\omega))}{\operatorname{Ker}(B / \beta(\omega))}\right]_{\hat{\omega}}\right),
$$

with discrete torsion $\hat{\omega}$ defined as in [9] and restricted to $\operatorname{Ker} B / \beta(\omega)$.

This result is more or less uniquely determined by previous results, for reasons already described. For example, suppose that $B$ itself is determined by some discrete torsion $\omega^{\prime}$. (This can happen, though will not always be the case.) Then the quantity

$$
B / \beta(\omega)=1 /\left(\beta\left(\omega^{\prime}\right) \beta(\omega)\right)=1 / \beta\left(\omega+\omega^{\prime}\right),
$$

and this prescription reduces to a special case of [9].

We will check this prediction explicitly in examples by comparing to genus-one partition functions, in sections 4.3.2, 4.4.2, 4.6.3. 
3. Suppose that $\iota^{*} \omega=0$ and $\omega=\pi^{*} \bar{\omega}$ for $\bar{\omega} \in H^{2}(G, \mathrm{U}(1))$. Then,

$$
\operatorname{QFT}\left([X / \Gamma]_{B, \omega}\right)=\operatorname{QFT}\left(\left[\frac{X \times \widehat{\operatorname{Coker} B}}{\operatorname{Ker} B}\right]_{\bar{\omega}+\hat{\omega}_{0}}\right),
$$

where $\hat{\omega}_{0}$ is the discrete torsion predicted in the case that $\omega$ is trivial.

This case is also more or less uniquely determined by consistency with previous results. We will check this prediction explicitly in examples by comparing to genus-one partition functions, in sections 4.2.2, 4.5.2.

We note that just as in our earlier work [9], to improve readability we unfortunately found it useful to mix additive and multiplicative notation.

Finally, in passing we note that a relation to theories realized as boundaries of higherdimensional theories is implicit in [48, prop. 4.243].

\section{Examples}

\subsection{Extension of cyclic groups to larger cyclic groups}

\subsubsection{Extension of $\mathbb{Z}_{2}$ to $\mathbb{Z}_{4}$}

In this section we will describe an example from [6] in the current language. In this example, one starts with a $G=\mathbb{Z}_{2}$ orbifold, and extend $G$ by $K=\mathbb{Z}_{2}$ (with trivial action on the space, but nontrivial action on $G$ twist fields) to $\Gamma=\mathbb{Z}_{4}$ :

$$
1 \longrightarrow \mathbb{Z}_{2} \longrightarrow \mathbb{Z}_{4} \longrightarrow \mathbb{Z}_{2} \longrightarrow 1
$$

(This example was also considered from a different perspective in [1, section 5.1.1].)

In this case, $H^{2}(\Gamma, \mathrm{U}(1))=0$, so we see that

$$
H^{1}\left(G, H^{1}(K, \mathrm{U}(1))\right)=\operatorname{Hom}\left(\mathbb{Z}_{2}, H^{1}\left(\mathbb{Z}_{2}, \mathrm{U}(1)\right)\right)=\operatorname{Hom}\left(\mathbb{Z}_{2}, \mathbb{Z}_{2}\right)=\mathbb{Z}_{2}
$$

injects into $H^{3}(G, \mathrm{U}(1))$, from (2.17). In particular, this means that the quantum symmetry phases are novel effects here, not captured by discrete torsion (which is of course absent for $\left.\mathbb{Z}_{4}\right)$.

In any event, there are two choices of quantum symmetry $B \in H^{1}\left(G, H^{1}(K, \mathrm{U}(1))\right)$, and we describe both cases below. First, consider the trivial case that $B=0$. In this case, Ker $B=G=\mathbb{Z}_{2}$ and Coker $B=K=\mathbb{Z}_{2}$, so from equation (3.2) we predict that

$$
\operatorname{QFT}\left(\left[X / \mathbb{Z}_{4}\right]\right)=\operatorname{QFT}\left(\coprod_{2}\left[X / \mathbb{Z}_{2}\right]\right)
$$

Next, consider the case that $B$ is the nontrivial element of $H^{1}\left(G, H^{1}(K, \mathrm{U}(1))\right)=\mathbb{Z}_{2}$. In this case, we have trivially $\operatorname{Ker} B=\operatorname{Coker} B=0$, so from equation (3.2) we predict that

$$
\operatorname{QFT}\left(\left[X / \mathbb{Z}_{4}\right]_{B}\right)=\operatorname{QFT}(X) .
$$


Now, let us compare to physics. The case that $B$ is trivial corresponds to ordinary decomposition, described in [16], and so will not be reviewed here. Let us turn to the case that $B$ is nontrivial. First, from the form of $B \in H^{1}\left(G, H^{1}(K, \mathrm{U}(1))\right)$ above, there is a quantum symmetry under which $x^{2}$ (the image of the generator of $K$ ) acts on the twist field associated with $x$ by $(-1)$, so that the genus-one $\mathbb{Z}_{4}$ orbifold partition functions obey

$$
x^{2} \underset{x^{n}}{\square}=(-)^{n}\left(1 \underset{x^{n}}{\square}\right),
$$

and more generally

$$
Z_{i, j}=(-)^{i} Z_{i, j-2}=(-)^{j} Z_{i-2, j}
$$

where $i, j \in\{0, \cdots, 3\}$. In particular,

$$
Z_{i, 2}=(-)^{i} Z_{i, 0}
$$

is equivalent to (4.5).

Taking into account these relationships and computing the entire genus-one partition function, we find

$$
\begin{aligned}
Z\left(\left[X / \mathbb{Z}_{4}\right]_{B}\right) & =\frac{1}{\left|\mathbb{Z}_{4}\right|} \sum_{g h=h g} Z_{g, h}, \\
& =\frac{1}{4}\left(Z_{0,0}+Z_{0,2}+Z_{2,0}+Z_{2,2}\right), \\
& =Z_{0,0}=Z(X),
\end{aligned}
$$

matching the prediction.

\subsubsection{Extension of $\mathbb{Z}_{2}$ by $\mathbb{Z}_{k}$}

This example is a variation on the previous one. Instead of extending the effectively-acting $\mathbb{Z}_{2}$ by another $\mathbb{Z}_{2}$, consider extending it by $\mathbb{Z}_{k}$ (that acts trivially on $X$ ) for $k \geq 2$ :

$$
1 \longrightarrow \mathbb{Z}_{k} \longrightarrow \mathbb{Z}_{2 k} \longrightarrow \mathbb{Z}_{2} \longrightarrow 1 \text {. }
$$

The possible values of the quantum symmetry $B$ are classified by

$$
H^{1}\left(G, H^{1}(K, \mathrm{U}(1))\right)=\operatorname{Hom}\left(\mathbb{Z}_{2}, \mathbb{Z}_{k}\right)=\left\{\begin{array}{cc}
1 & k \text { odd } \\
\mathbb{Z}_{2} & k \text { even }
\end{array}\right.
$$

In the case that the quantum symmetry is trivial, Ker $B=\mathbb{Z}_{2}$, Coker $B=K=\mathbb{Z}_{k}$, and from ordinary decomposition [16],

$$
\operatorname{QFT}\left(\left[X / \mathbb{Z}_{2 k}\right]\right)=\operatorname{QFT}\left(\coprod_{k}\left[X / \mathbb{Z}_{2}\right]\right) .
$$

Next, consider the case that $B$ is nontrivial - for which we also assume that $k$ is even. In this case, Ker $B=0$, and Coker $B=\mathbb{Z}_{k / 2}$. Thus, from equation (3.2), we predict that

$$
\operatorname{QFT}\left(\left[X / \mathbb{Z}_{2 k}\right]_{B}\right)=\operatorname{QFT}\left(\coprod_{k / 2} X\right) \text {. }
$$


Now, let us check this prediction in physics. We only consider the case that the quantum symmetry $B$ is nontrivial (and also that $k$ is even), as the case $B$ trivial corresponds to ordinary decomposition. If we let $x$ denote the generator of $\mathbb{Z}_{2 k}$, so that $x^{2}$ generates the image of $K$, then the quantum symmetry acts in the form

$$
x^{2} \underset{x}{\square}=-(1 \underset{x}{\square})
$$

or more generally,

$$
Z_{i, j}=(-)^{i} Z_{i, j-2}=(-)^{j} Z_{i-2, j},
$$

where $i, j \in\{0, \cdots, 2 k-1\}$. In this example, this is the concrete meaning of $B$.

Taking into account these relationships and computing the entire genus-one partition function, we find

$$
\begin{aligned}
Z\left(\left[X / \mathbb{Z}_{2 k}\right]_{B}\right) & =\frac{1}{\left|\mathbb{Z}_{2 k}\right|} \sum_{g h=h g} Z_{g, h}, \\
& =\frac{1}{2 k} \sum_{i, j=0}^{k-1} Z_{2 i, 2 j}, \\
& =\frac{1}{2 k}(k)^{2} Z_{0,0}=\frac{k}{2} Z(X), \\
& =Z\left(\coprod_{k / 2} X\right),
\end{aligned}
$$

matching the prediction (4.14).

Another way to understand this problem is via decomposition $[9,16,35,36]$. For $k$ even, as we have assumed, the orbifold group $\mathbb{Z}_{2 k}$ can be described as an extension of $\mathbb{Z}_{4}$ by $\mathbb{Z}_{k / 2}$ :

$$
1 \longrightarrow \mathbb{Z}_{k / 2} \longrightarrow \mathbb{Z}_{2 k} \longrightarrow \mathbb{Z}_{4} \longrightarrow 1 \text {. }
$$

The $\mathbb{Z}_{k / 2}$ acts trivially on both the space $X$ as well as the twisted sectors - the former because it is a subgroup of the $\mathbb{Z}_{k}$ that acted trivially on $X$, the latter because the action on twisted sectors was encoded in $\mathbb{Z}_{2}$ subgroup which $\mathbb{Z}_{k / 2}$ has quotiented out.

Then, applying decomposition $[9,16,35,36]$, we have immediately that

$$
\operatorname{QFT}\left(\left[X / \mathbb{Z}_{2 k}\right]_{B}\right)=\operatorname{QFT}\left(\left[\frac{X \times \hat{\mathbb{Z}}_{k / 2}}{\mathbb{Z}_{4}}\right]_{B}\right)=\operatorname{QFT}\left(\coprod_{k / 2}\left[X / \mathbb{Z}_{4}\right]_{B}\right),
$$

and as we know that

$$
\operatorname{QFT}\left(\left[X / \mathbb{Z}_{4}\right]_{B}\right)=X
$$

decomposition therefore tells us that

$$
\operatorname{QFT}\left(\left[X / \mathbb{Z}_{2 k}\right]_{B}\right)=\operatorname{QFT}\left(\coprod_{k / 2} X\right)
$$

in agreement with the prediction (4.14) and also with physics results in this case. 


\subsubsection{Extension of $\mathbb{Z}_{3}$ to $\mathbb{Z}_{9}$}

In this section we start with a $G=\mathbb{Z}_{3}$ orbifold, extend $G$ by $K=\mathbb{Z}_{3}$ to $\Gamma=\mathbb{Z}_{9}$ :

$$
1 \longrightarrow \mathbb{Z}_{3} \longrightarrow \mathbb{Z}_{9} \longrightarrow \mathbb{Z}_{3} \longrightarrow 1 \text {. }
$$

In this case,

$$
B \in \operatorname{Hom}\left(G, H^{1}(K, \mathrm{U}(1))=\mathbb{Z}_{3} .\right.
$$

Our prediction (section 3) falls into the following cases:

1. In the case $B$ is trivial, this reduces to ordinary decomposition, for which

$$
\operatorname{QFT}([X / \Gamma])=\operatorname{QFT}\left(\left[\frac{X \times \hat{K}}{G}\right]\right)=\operatorname{QFT}\left(\coprod_{3}\left[X / \mathbb{Z}_{3}\right]\right) \text {. }
$$

2. In both of the cases that $B$ is nontrivial, $\operatorname{Ker} B=0$ and Coker $B=0$, so we predict (section 3) that

$$
\operatorname{QFT}\left([X / \Gamma]_{B}\right)=\operatorname{QFT}(X) .
$$

We can confirm these predictions explicitly at the level of partition functions. The analysis for the case $B$ is trivial is standard, and will not be repeated here. Suppose $B$ is nontrivial.

- Consider genus-one $\Gamma$-twisted sectors which project to

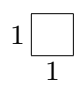

in the $G$ orbifold. Phases are various powers of $B(1, z)$ for $z \in K$, but for all $z$, $B(1, z)=1$. Thus, the sector above appears with multiplicity $|K|^{2}=9$.

- Consider, for example, genus-one $\Gamma$-twisted sectors which project to the $G$-twisted sector

$$
1 \square_{g}
$$

for $g \neq 1$ an element of $G=\mathbb{Z}_{3}$. The different $\Gamma$-twisted sectors which project to this sector are weighted by different roots of unity, and the sum over such sectors collapses to copies of sums over roots of unity, which vanishes. Therefore, there are no net contributions to the $\Gamma$-orbifold partition function from these sectors, and similarly one can show that there are no net contributions from any sectors that do not project to the trivial sector.

Summarizing, the genus-one partition function is given by

$$
\begin{aligned}
Z\left([X / \Gamma]_{B}\right) & =\frac{1}{\Gamma} \sum_{g, h} g \square_{h}, \\
& =\frac{|K|^{2}}{|\Gamma|}(1 \square)=1 \square_{1}, \\
& =Z(X),
\end{aligned}
$$

confirming the prediction. 


\section{$4.2 \quad$ Extension of $\mathbb{Z}_{2} \times \mathbb{Z}_{2}$ to $\mathbb{Z}_{2} \times \mathbb{Z}_{4}$}

In this section we begin with a $G=\mathbb{Z}_{2} \times \mathbb{Z}_{2}$ orbifold, extended by $K=\mathbb{Z}_{2}$ to $\Gamma=\mathbb{Z}_{2} \times \mathbb{Z}_{4}$. We write $G=\langle\bar{a}, \bar{b}\rangle$, where $\bar{a}^{2}=1=\bar{b}^{2}$, and $\Gamma=\langle a, b\rangle$, where $\bar{a}=a K$ and $\bar{b}=b K$, with $K=\left\langle b^{2}\right\rangle$.

Now, the quantum symmetry is defined by an element $B \in H^{1}\left(G, H^{1}(K, \mathrm{U}(1))\right)$, which is defined by its action on the generators $\bar{a}, \bar{b}$. We will systematically study the orbifold $[X / \Gamma]$ for all choices of $B$, initially without and later with discrete torsion, and compare with the predictions of section 3 .

First, the possible values for $B$ are as follows: ${ }^{4}$

1. $B(\bar{a})=+1, B(\bar{b})=+1$ (the trivial case),

2. $B(\bar{a})=-1, B(\bar{b})=+1$,

3. $B(\bar{a})=+1, B(\bar{b})=-1$,

4. $B(\bar{a})=-1, B(\bar{b})=-1$.

We will first analyze orbifolds without additional discrete torsion $\omega \in H^{2}(\Gamma, \mathrm{U}(1))$, then separately study the effect of adding $\omega \in H^{2}(\Gamma, \mathrm{U}(1))$.

\subsubsection{Without discrete torsion}

For each possible $B$, we make a prediction for the structure of the orbifold $[X / \Gamma]$ using the conjecture of section 3 , as follows:

1. First, consider the case that $B$ is trivial: $B(\bar{a})=+1$ and $B(\bar{b})=+1$. In this case, the $[X / \Gamma]$ orbifold can be understood via decomposition $[9,16,35,36]$, which predicts

$$
\operatorname{QFT}([X / \Gamma])=\operatorname{QFT}\left(\coprod_{2}\left[X / \mathbb{Z}_{2} \times \mathbb{Z}_{2}\right]\right)
$$

a disjoint union of two copies of $\left[X / \mathbb{Z}_{2} \times \mathbb{Z}_{2}\right]$. For essentially the same reasons as $[9$, section 6.1], since the extension is a pullback from

$$
1 \longrightarrow \mathbb{Z}_{2} \longrightarrow \mathbb{Z}_{4} \longrightarrow \mathbb{Z}_{2} \longrightarrow 1
$$

neither copy has discrete torsion.

2. Next, consider the case that $B(\bar{a})=-1$ and $B(\bar{b})=+1$. Here, Coker $B=0$ and Ker $B=\mathbb{Z}_{2}=\langle\bar{b}\rangle$, so from section 3 we predict that

$$
\operatorname{QFT}\left([X / \Gamma]_{B}\right)=\operatorname{QFT}\left(\left[X / \mathbb{Z}_{2}=\langle\bar{b}\rangle\right]\right) .
$$

\footnotetext{
${ }^{4}$ Here, and in many instances below, we are identifying $H^{1}\left(\mathbb{Z}_{2}, \mathrm{U}(1)\right) \cong \operatorname{Hom}\left(\mathbb{Z}_{2}, \mathrm{U}(1)\right) \cong \mathbb{Z}_{2}=\{+1,-1\}$. So for the case at hand, with $K=\left\{1, b^{2}\right\}$, if we write $B(\bar{a})=-1$, we mean that

$$
B(\bar{a})(1)=1, \quad B(\bar{a})\left(b^{2}\right)=-1 .
$$
}


3. Next, consider the case that $B(\bar{a})=+1$ and $B(\bar{b})=-1$. Here, Coker $B=0$ and Ker $B=\mathbb{Z}_{2}=\langle\bar{a}\rangle$, so we predict that

$$
\operatorname{QFT}\left([X / \Gamma]_{B}\right)=\operatorname{QFT}\left(\left[X / \mathbb{Z}_{2}=\langle\bar{a}\rangle\right]\right) .
$$

4. Finally, consider the case that $B(\bar{a})=-1$ and $B(\bar{b})=-1$. Here, Coker $B=0$ and Ker $B=\langle\bar{a} \bar{b}\rangle$, so we predict that

$$
\operatorname{QFT}\left([X / \Gamma]_{B}\right)=\operatorname{QFT}\left(\left[X / \mathbb{Z}_{2}=\langle\bar{a} \bar{b}\rangle\right]\right) .
$$

Finally, we check the predictions in each case by computing genus-one partition functions.

1. First, consider the case that $B$ is trivial: $B(\bar{a})=+1$ and $B(\bar{b})=+1$. Essentially this case was discussed in $[9$, section 6.1$]$, where it was argued that the genus-one partition function

$$
Z([X / \Gamma])=Z\left(\coprod_{2}\left[X / \mathbb{Z}_{2} \times \mathbb{Z}_{2}\right]\right) .
$$

2. Next, consider the case $B(\bar{a})=-1$ and $B(\bar{b})=+1$. The center $K$ acts nontrivially on twisted sectors twisted by $a$, for example,

$$
b^{2} \square_{a}=-\left(1 \square_{a}\right), \quad b^{2} \square_{b}=+\left(1 \square_{b}^{\square}\right) .
$$

Using relations of this form, it is straightforward to check that the genus-one partition function obeys

$$
\begin{aligned}
Z\left([X / \Gamma]_{B}\right) & =\frac{1}{|\Gamma|} \sum_{g h=h g} g \square_{h}, \\
& =\frac{4}{8}\left[1 \square_{1}+1 \underset{\bar{b}}{\square}+\bar{b} \square_{1}+\bar{b} \square_{\bar{b}}\right] \\
& =Z\left(\left[X / \mathbb{Z}_{2}=\langle\bar{b}\rangle\right]\right)
\end{aligned}
$$

matching the prediction.

3. Next, consider the case $B(\bar{a})=+1$ and $B(\bar{b})=-1$. Here, the center $K$ acts nontrivially on twisted sectors twisted by $b$, for example,

$$
b^{2} \square_{a}=+\left(1 \square_{a}\right), \quad b^{2} \square_{b}=-\left(1 \square_{b}\right) .
$$

Using relations of this form, it is straightforward to check that the genus-one partition function obeys

$$
\begin{aligned}
Z\left([X / \Gamma]_{B}\right) & =\frac{1}{|\Gamma|} \sum_{g h=h g} g \square_{h}, \\
& =\frac{4}{8}\left[1 \square_{1}+1 \underset{\bar{a}}{\square}+\bar{a} \underset{1}{\square}+\bar{a} \frac{\square}{\bar{a}}\right], \\
& =Z\left(\left[X / \mathbb{Z}_{2}=\langle\bar{a}\rangle\right]\right),
\end{aligned}
$$

matching the prediction. 
4. Finally, consider the case $B(\bar{a})=-1$ and $B(\bar{b})=-1$. Here, the center $K$ acts nontrivially on twisted sectors twisted by either $a$ or $b$, for example,

$$
b^{2} \underset{a}{\square_{a}}=-(1 \underset{a}{\square}), \quad b^{2} \underset{b}{\square}=-(1 \underset{b}{\square}), \quad b^{2} \underset{a b}{\square_{b}}=+(1 \underset{a b}{\square}) .
$$

Using relations of this form, it is straightforward to check that the genus-one partition function obeys

$$
\begin{aligned}
Z\left([X / \Gamma]_{B}\right) & =\frac{1}{|\Gamma|} \sum_{g h=h g} g \square_{h}, \\
& =\frac{4}{8}\left[1 \square_{1}+1 \underset{\bar{a} \bar{b}}{\square}+\bar{a} \bar{b} \overline{\square_{1}}+\bar{a} \bar{b} \bar{\square} \square_{\bar{a} \bar{b}}\right] \\
& =Z\left(\left[X / \mathbb{Z}_{2}=\langle\bar{a} \bar{b}\rangle\right]\right),
\end{aligned}
$$

matching the prediction.

\subsubsection{With discrete torsion}

So far we have considered the $[X / \Gamma]$ orbifold without discrete torsion. Now, from $[9$, section D.2], we know that $H^{2}\left(\mathbb{Z}_{2} \times \mathbb{Z}_{4}, \mathrm{U}(1)\right)=\mathbb{Z}_{2}$, so one can turn on one nontrivial element of discrete torsion in the $\Gamma=\mathbb{Z}_{2} \times \mathbb{Z}_{4}$ orbifold. We do so next, denoting by $\omega$ the nontrivial element of $H^{2}(\Gamma, \mathrm{U}(1))$. Letting $\iota: K \hookrightarrow \Gamma$ denote the inclusion and $\pi: \Gamma \rightarrow G$ the projection, then as observed in [9, section 6.1], $\iota^{*} \omega=0$ and $\omega=\pi^{*} \bar{\omega}$, for $\bar{\omega}$ the nontrivial element of $H^{2}\left(\mathbb{Z}_{2} \times \mathbb{Z}_{2}, \mathrm{U}(1)\right)$. This will play an important role in applying section 3 .

Next, we will make predictions for each of the four cases above, using the methods of section 3 .

1. First, consider the case that $B$ is trivial, $B(\bar{a})=+1$ and $B(\bar{b})=+1$. This case was studied in $[9$, section 6.1$]$, which made (and confirmed) the prediction

$$
\operatorname{QFT}\left([X / \Gamma]_{\omega}\right)=\operatorname{QFT}\left(\coprod_{2}\left[X / \mathbb{Z}_{2} \times \mathbb{Z}_{2}\right]_{\bar{\omega}}\right) .
$$

2. The remaining three cases are identical to the cases studied in the $\Gamma$ orbifold without discrete torsion. For each $B$, Ker $B=\mathbb{Z}_{2}$, which does not admit discrete torsion, so the predicted orbifold is the same.

Finally, we check each of these predictions by computing genus-one partition functions.

1. First, consider the case that $B$ is trivial, $B(\bar{a})=+1$ and $B(\bar{b})=+1$. This case was studied in $[9$, section 6.1$]$, where it was shown that

$$
Z\left([X / \Gamma]_{\omega}\right)=Z\left(\coprod_{2}\left[X / \mathbb{Z}_{2} \times \mathbb{Z}_{2}\right]_{\bar{\omega}}\right) .
$$


2. Next, consider the case that $B(\bar{a})=-1$ and $B(\bar{b})=+1$. Here, the center $K$ acts nontrivially on twisted sectors twisted by $a$. The genus-one partition function is given by

$$
\begin{aligned}
Z\left([X / \Gamma]_{\omega}\right) & =\frac{1}{|\Gamma|} \sum_{g h=h g} \epsilon(g, h)\left(g \square_{h}\right) \\
& =\frac{4}{8}\left[1 \square_{1}+1 \square_{\bar{b}}+\bar{b} \square_{1}+\bar{b} \square_{\bar{b}}\right] \\
& =Z\left(\left[X / \mathbb{Z}_{2}=\langle\bar{b}\rangle\right]\right)
\end{aligned}
$$

matching the prediction.

3. Next, consider the case that $B(\bar{a})=+1$ and $B(\bar{b})=-1$. The center $K$ acts nontrivially on twisted sectors twisted by $b$. The genus-one partition function is given by

$$
\begin{aligned}
Z\left([X / \Gamma]_{\omega}\right) & =\frac{1}{|\Gamma|} \sum_{g h=h g} \epsilon(g, h)(g \underset{h}{\square}) \\
& =\frac{4}{8}\left[1 \underset{1}{\square}+1 \underset{\bar{a}}{\square}+\bar{a} \underset{1}{\square}+\bar{a} \square_{\bar{a}}\right] \\
& =Z\left(\left[X / \mathbb{Z}_{2}=\langle\bar{a}\rangle\right]\right)
\end{aligned}
$$

matching the prediction.

4. Finally, consider the case $B(\bar{a})=-1$ and $B(\bar{b})=-1$. Here, the center $K$ acts nontrivially on twisted sectors twisted by either $a$ or $b$. The genus-one partition function is given by

$$
\begin{aligned}
Z\left([X / \Gamma]_{\omega}\right) & =\frac{1}{|\Gamma|} \sum_{g h=h g} \epsilon(g, h)\left(g \square_{h}\right) \\
& =\frac{4}{8}\left[1 \underset{1}{1 \square}+{ }_{\bar{a} \bar{b}}+\bar{a} \bar{b} \bar{\square}\right. \\
& +\bar{a} \bar{b} \bar{\square} \square] \\
& =Z\left(\left[X / \mathbb{Z}_{2}=\langle\bar{a} \bar{b}\rangle\right]\right),
\end{aligned}
$$

matching the prediction.

\subsubsection{Summary}

We summarize the results of this analysis in table 1 .

\subsection{Extension of $\mathbb{Z}_{2} \times \mathbb{Z}_{2}$ to $D_{4}$}

In this section we consider a $G=\mathbb{Z}_{2} \times \mathbb{Z}_{2}$ theory, generated by $\bar{a}$ and $\bar{b}$ of order two, extended to $\Gamma=D_{4}$ :

$$
1 \longrightarrow \mathbb{Z}_{2} \longrightarrow D_{4} \longrightarrow \mathbb{Z}_{2} \times \mathbb{Z}_{2} \longrightarrow 1
$$




\begin{tabular}{c|cc|cc}
\hline Case & $B(\bar{a})$ & $B(\bar{b})$ & Without discrete torsion & With discrete torsion \\
\hline 1 & +1 & +1 & {$\left[X / \mathbb{Z}_{2} \times \mathbb{Z}_{2}\right] \amalg\left[X / \mathbb{Z}_{2} \times \mathbb{Z}_{2}\right]$} & {$\left[X / \mathbb{Z}_{2} \times \mathbb{Z}_{2}\right]_{\text {d.t. }} \amalg\left[X / \mathbb{Z}_{2} \times \mathbb{Z}_{2}\right]_{\text {d.t. }}$} \\
2 & -1 & +1 & {$\left[X / \mathbb{Z}_{2}=\langle\bar{b}\rangle\right]$} & {$\left[X / \mathbb{Z}_{2}=\langle\bar{b}\rangle\right]$} \\
3 & +1 & -1 & {$\left[X / \mathbb{Z}_{2}=\langle\bar{a}\rangle\right]$} & {$\left[X / \mathbb{Z}_{2}=\langle\bar{a}\rangle\right]$} \\
4 & -1 & -1 & {$\left[X / \mathbb{Z}_{2}=\langle\bar{a} \bar{b}\rangle\right]$} & {$\left[X / \mathbb{Z}_{2}=\langle\bar{a} \bar{b}\rangle\right]$} \\
\hline
\end{tabular}

Table 1. Summary of results for $\mathbb{Z}_{2} \times \mathbb{Z}_{4}$ orbifold extending $\mathbb{Z}_{2} \times \mathbb{Z}_{2}$ orbifold.

where $\Gamma=D_{4}$ is the dihedral group of order 8 , and $K=\mathbb{Z}_{2}$. We will write the elements of $D_{4}$ as

$$
\{1, z, a, b, a z, b z, a b, b a=a b z\}
$$

where $a^{2}=b^{4}=1, b^{2}=z, z$ is central (generating the image of $K$ ), a projects to $\bar{a}$, and $b$ projects to $\bar{b}$. (This example was also considered from a different perspective in $[1$, section 5.2.1].)

The quantum symmetry $B$ is an element of

$$
H^{1}\left(G, H^{1}(K, \mathrm{U}(1))=\operatorname{Hom}\left(\mathbb{Z}_{2} \times \mathbb{Z}_{2}, \hat{\mathbb{Z}}_{2}\right) .\right.
$$

Any nontrivial $B$ is necessarily surjective, hence $\operatorname{Ker} B=\mathbb{Z}_{2}$ and Coker $B=0$. From the general conjecture (3.2), we predict that

$$
\operatorname{QFT}\left([X / \Gamma]_{B}\right)=\operatorname{QFT}\left(\left[X / \mathbb{Z}_{2}\right]\right)
$$

for any nontrivial $B$, with the choice of $\mathbb{Z}_{2} \subset \mathbb{Z}_{2} \times \mathbb{Z}_{2}$ depending upon the choice of $B$. If $B$ is trivial, then we have

$$
\operatorname{QFT}[[X / \Gamma])=\operatorname{QFT}\left(\left[\frac{X \times \hat{K}}{\mathbb{Z}_{2} \times \mathbb{Z}_{2}}\right]_{\hat{\omega}}\right)=\operatorname{QFT}\left(\left[X / \mathbb{Z}_{2} \times \mathbb{Z}_{2}\right] \coprod\left[X / \mathbb{Z}_{2} \times \mathbb{Z}_{2}\right]_{\text {d.t. }}\right),
$$

as explored in detail in [16, section 5.2].

To make this more precise, let us consider the four possible values of $B \in H^{1}\left(G, H^{1}\right.$ $(K, \mathrm{U}(1)))$, and the physical meaning of each. We can enumerate the possibilities as follows:

1. $B(\bar{a})=+1, B(\bar{b})=+1$,

2. $B(\bar{a})=-1, B(\bar{b})=+1$,

3. $B(\bar{a})=+1, B(\bar{b})=-1$,

4. $B(\bar{a})=-1, B(\bar{b})=-1$.

We will first consider cases without discrete torsion, then add discrete torsion $\omega \in$ $H^{2}(\Gamma, \mathrm{U}(1))$. 


\subsubsection{Without discrete torsion}

1. First, consider the case in which

$$
B(\bar{a})=+1=B(\bar{b}) .
$$

This orbifold can be understood in terms of decomposition [9, 16, 35, 36], which predicts that

$$
\operatorname{QFT}\left(\left[X / D_{4}\right]\right)=\operatorname{QFT}\left(\left[X / \mathbb{Z}_{2} \times \mathbb{Z}_{2}\right] \coprod\left[X / \mathbb{Z}_{2} \times \mathbb{Z}_{2}\right]_{\text {d.t. }}\right),
$$

where the second $\mathbb{Z}_{2} \times \mathbb{Z}_{2}$ orbifold has discrete torsion. (See [16, section 5.2] for further analyses of this example.)

2. Next, consider the second case, in which $B(\bar{a})=-1$ and $B(\bar{b})=+1$. Here, Ker $B=\langle\bar{b}\rangle$, so we predict that

$$
\operatorname{QFT}\left([X / \Gamma]_{B}\right)=\operatorname{QFT}\left(\left[X / \mathbb{Z}_{2}=\langle\bar{b}\rangle\right]\right) .
$$

Now, we check that prediction at the level of partition functions. The center $K$ acts trivially on the space but nontrivially on twisted sectors twisted by $a$, as dictated by B. For example:

$$
\begin{aligned}
& z \underset{b}{\square}=+(1 \underset{b}{\square}), \quad b z \underset{b}{\square}=+(b \underset{b}{\square}), \quad b z \underset{b z}{\square}=+\left(b \frac{\square}{b z}\right), \\
& z \underset{a}{\square}=-(1 \underset{a}{\square}), \quad z \underset{a b}{\square}=-(1 \underset{a b}{\square}), \quad z \underset{a b z}{\square}=-(1 \underset{a b z}{\square}),
\end{aligned}
$$

and so forth.

For this $B$, it is straightforward to compute the genus-one partition function

$$
\begin{aligned}
Z\left(\left[X / D_{4}\right]\right) & =\frac{1}{\left|D_{4}\right|} \sum_{g h=h g} g \square_{h}, \\
& =\frac{4}{8}\left[1 \square_{1}+1 \square_{\bar{b}}+\bar{b} \underset{1}{\square}+\bar{b} \square_{\bar{b}}\right]=Z\left(\left[X / \mathbb{Z}_{2}\right]\right),
\end{aligned}
$$

a $\mathbb{Z}_{2}=\langle\bar{b}\rangle$ orbifold, agreeing with the prediction.

3. Next, consider the third case, in which $B(\bar{a})=+1$ and $B(\bar{b})=-1$. Here, Ker $B=\langle\bar{a}\rangle$, so we predict that

$$
\operatorname{QFT}\left([X / \Gamma]_{B}\right)=\operatorname{QFT}\left(\left[X / \mathbb{Z}_{2}=\langle\bar{a}\rangle\right]\right) .
$$

Now, we check that prediction at the level of partition functions. The center $K$ acts trivially on the space, but nontrivially on twisted sectors twisted by $b$, as dictated by $B$, for example:

$$
\begin{aligned}
& z \underset{b}{\square_{b}}=-(1 \underset{b}{\square}), \quad b z \underset{b}{\square}=-(b \underset{b}{b}), \quad b z_{b z}=-(b \underset{b z}{\square}), \\
& z \underset{a}{\square}=+(1 \underset{a}{\square}), \quad z \underset{a b z}{\square}=-(1 \underset{a b z}{\square}),
\end{aligned}
$$

and so forth. 
For this $B$, it is now straightforward to compute the genus-one partition function:

$$
\begin{aligned}
Z\left(\left[X / D_{4}\right]\right) & =\frac{1}{\left|D_{4}\right|} \sum_{g h=h g} g \square_{h}, \\
& =\frac{1}{8}\left[1 \square_{1}+z \square_{1}+1 \square_{z}+z \square_{z}+1 \square_{a}+\cdots+a z \square_{a z}\right], \\
& =\frac{4}{8}\left[1 \square_{1}+1 \square_{\bar{a}}+\bar{a} \frac{\square}{1}+\bar{a} \square_{\bar{a}}\right]=Z\left(\left[X / \mathbb{Z}_{2}\right]\right) .
\end{aligned}
$$

All sectors involving a " $b$ " have cancelled out, and the theory is equivalent to a $\mathbb{Z}_{2}$ orbifold by $\mathbb{Z}_{2}=\langle\bar{a}\rangle$, agreeing with the prediction.

4. Next, consider the fourth case, in which $B(\bar{a})=-1$ and $B(\bar{b})=-1$. Here, Ker $B=\langle\bar{a} \bar{b}\rangle$, so we predict that

$$
\operatorname{QFT}\left([X / \Gamma]_{B}\right)=\operatorname{QFT}\left(\left[X / \mathbb{Z}_{2}=\langle\bar{a} \bar{b}\rangle\right]\right)
$$

Now, we check that prediction at the level of partition functions. In this case, $B$ dictates the relations

$$
\begin{aligned}
& z \underset{b}{\square}=-(1 \underset{b}{1 \square}), \quad b z \underset{b}{\square}=-(b \underset{b}{b}), b z \underset{b z}{\square}=-(b \underset{b z}{\square}), \\
& z \square_{a}=-(1 \underset{a}{\square}), \quad z \underset{a b}{\square}=+(1 \underset{a b}{\square}), \quad z \underset{a b z}{\square_{a b}}=+\left(1 \square_{a b z}^{\square}\right),
\end{aligned}
$$

and so forth.

For this $B$, it is straightforward to compute the genus-one partition function:

$$
\begin{aligned}
Z\left(\left[X / D_{4}\right]\right) & =\frac{1}{\left|D_{4}\right|} \sum_{g h=h g} g \square_{h}, \\
& =\frac{4}{8}\left[1 \underset{1}{\square}+1 \underset{\bar{a} \bar{b}}{\square_{1}}+\bar{a} \bar{b} \bar{\square}\right.
\end{aligned}
$$

This is a $\mathbb{Z}_{2}=\langle\bar{a} \bar{b}\rangle$ orbifold, agreeing with the prediction.

\subsubsection{With discrete torsion}

Now, the $\Gamma=D_{4}$ orbifold admits one possible nonzero value of discrete torsion, as $[9$, appendix D.3]

$$
H^{2}\left(D_{4}, \mathrm{U}(1)\right)=\mathbb{Z}_{2}
$$

Before describing the result of turning on that discrete torsion, let us walk through its analysis formally. A $D_{4}$ orbifold with trivially-acting central $\mathbb{Z}_{2}$ and discrete torsion was studied in [9, section 5.5], and in the notation of that reference, $\iota^{*} \omega=0$ but $\beta(\omega) \neq 0$.

Now, $\beta(\omega) \in H^{1}\left(G, H^{1}(K, \mathrm{U}(1))\right)$ where in this case, $G=D_{4} / \mathbb{Z}_{2}=\mathbb{Z}_{2} \times \mathbb{Z}_{2}$. For a general $\omega \in H^{2}(\Gamma, \mathrm{U}(1))$, one defines [9]

$$
\beta(\omega)(q, k)=\frac{\omega\left(k s(q), s(q)^{-1}\right)}{\omega\left(s(q)^{-1}, k s(q)\right)},
$$


where $s: G \rightarrow \Gamma$ is a section, which here we can take to be

$$
s(1)=1, \quad s(\bar{a})=a, \quad s(\bar{b})=b, \quad s(\bar{a} \bar{b})=a b,
$$

for which one computes the nontrivial cases [9, section 5.5]

$$
\beta(\omega)(\bar{a}, z)=-1, \quad \beta(\omega)(\bar{b}, z)=+1, \quad \beta(\omega)(\bar{a} \bar{b}, z)=-1 .
$$

(As noted in section 2.3, $\beta(\omega)$ is independent of the choice of section s.)

The effect of $\beta(\omega)$, it should now be clear, is to modify the map $B$, essentially by flipping its action on $a$-twisted sectors. We shall see this explicitly next.

Next, we systematically describe the results for $\left[X / D_{4}\right]$ orbifolds with discrete torsion, in all cases.

1. We begin with the first case, with trivial $B$. This is an example of decomposition with discrete torsion, described in [9, section 5.5]. The result is

$$
\operatorname{QFT}\left(\left[X / D_{4}\right]_{\text {d.t. }}\right)=\operatorname{QFT}\left(\left[X / \mathbb{Z}_{2}\right]\right),
$$

where the $\mathbb{Z}_{2}$ is generated by $\bar{b}$.

2. Next, consider the case $B(\bar{a})=-1, B(\bar{b})=+1$. In this case, $B / \beta(\omega)$ is trivial, so we predict

$$
\operatorname{QFT}\left([X / \Gamma]_{B, \omega}\right)=\operatorname{QFT}\left(\coprod_{2}\left[X / \mathbb{Z}_{2} \times \mathbb{Z}_{2}\right]_{\hat{\omega}}\right),
$$

for some discrete torsion $\hat{\omega}$ on the components.

Next, we check the prediction at the level of partition functions. Here, it is straightforward to compute

$$
\begin{aligned}
& Z\left(\left[X / D_{4}\right]_{\text {d.t. }}\right)=\frac{1}{\left|D_{4}\right|} \sum_{g h=h g} \epsilon(g, h)(g \square), \\
& =\frac{4}{8}\left[1 \square_{1}+1 \square_{\bar{a}}+1 \square_{\bar{b}}+1 \square_{\bar{a} \bar{b}}+\bar{a} \square_{1}+\bar{a} \square_{\bar{a}}+\bar{b} \square_{\overline{1}}+\bar{b} \square_{\bar{b}}\right. \\
& +\bar{a} \bar{b} \underset{1}{\square}+\bar{a} \bar{b} \bar{\square}[\bar{a} \bar{b}] \text {, } \\
& =\left[X / D_{4}\right]=\left[X / \mathbb{Z}_{2} \times \mathbb{Z}_{2}\right] \coprod\left[X / \mathbb{Z}_{2} \times \mathbb{Z}_{2}\right]_{\text {d.t. }} .
\end{aligned}
$$

Thus, this case is equivalent to the original $D_{4}$ orbifold, agreeing with our prediction.

3. Next, consider the third case, $B(\bar{a})=+1, B(\bar{b})=-1$. In this case, $(B / \beta(\omega))(\bar{a})=-1$, $(B / \beta(\omega))(\bar{b})=-1$, so that $\operatorname{Ker} B / \beta(\omega)=\langle\bar{a} \bar{b}\rangle$, with no cokernel, hence we predict

$$
\operatorname{QFT}\left([X / \Gamma]_{B, \omega}\right)=\operatorname{QFT}\left(\left[X / \mathbb{Z}_{2}=\langle\bar{a} \bar{b}\rangle\right]\right) .
$$




\begin{tabular}{c|cc|cc}
\hline Case & $B(\bar{a})$ & $B(\bar{b})$ & Without discrete torsion & With discrete torsion \\
\hline 1 & +1 & +1 & {$\left[X / \mathbb{Z}_{2} \times \mathbb{Z}_{2}\right] \amalg\left[X / \mathbb{Z}_{2} \times \mathbb{Z}_{2}\right]_{\text {d.t. }}$} & {$\left[X / \mathbb{Z}_{2}=\langle\bar{b}\rangle\right]$} \\
2 & -1 & +1 & {$\left[X / \mathbb{Z}_{2}=\langle\bar{b}\rangle\right]$} & {$\left[X / \mathbb{Z}_{2} \times \mathbb{Z}_{2}\right] \amalg\left[X / \mathbb{Z}_{2} \times \mathbb{Z}_{2}\right]_{\text {d.t. }}$} \\
3 & +1 & -1 & {$\left[X / \mathbb{Z}_{2}=\langle\bar{a}\rangle\right]$} & {$\left[X / \mathbb{Z}_{2}=\langle\bar{a} \bar{b}\rangle\right]$} \\
4 & -1 & -1 & {$\left[X / \mathbb{Z}_{2}=\langle\bar{a} \bar{b}\rangle\right]$} & {$\left[X / \mathbb{Z}_{2}=\langle\bar{a}\rangle\right]$} \\
\hline
\end{tabular}

Table 2. Summary of results for $D_{4}$ orbifold extending $\mathbb{Z}_{2} \times \mathbb{Z}_{2}$ orbifold.

Now, we will check that prediction. The genus-one partition function is given by

$$
\begin{aligned}
Z\left(\left[X / D_{4}\right]_{\text {d.t. }}\right) & =\frac{1}{\left|D_{4}\right|} \sum_{g h=h g} \epsilon(g, h)\left(g \square_{h}\right) \\
& =\frac{4}{8}\left[1 \square_{1}+1{ }_{\bar{a} \bar{b}}+\bar{a} \bar{b}{ }_{1}+\bar{a} \bar{b} \square_{\bar{a} \bar{b}}\right]=Z\left(\left[X / \mathbb{Z}_{2}\right]\right) .
\end{aligned}
$$

Thus, this case is equivalent to a $\mathbb{Z}_{2}=\langle\bar{a} \bar{b}\rangle$ orbifold, agreeing with our prediction.

4. Now, consider the fourth case. Here, $(B / \beta(\omega))(\bar{a})=+1,(B / \beta(\omega))(\bar{b}=-1$, hence Ker $B / \beta(\omega)=\langle\bar{a}\rangle$, with no cokernel, and so we predict

$$
\operatorname{QFT}\left([X / \Gamma]_{B, \omega}\right)=\operatorname{QFT}\left(\left[X / \mathbb{Z}_{2}=\langle\bar{a}\rangle\right]\right) .
$$

Next, we check the prediction. The genus-one partition function is given by

$$
\begin{aligned}
Z\left(\left[X / D_{4}\right]_{\text {d.t. }}\right) & =\frac{1}{\left|D_{4}\right|} \sum_{g h=h g} \epsilon(g, h)\left(g \square_{h}\right) \\
& =\frac{4}{8}\left[1 \square_{1}+1 \underset{\bar{a}}{\square}+\bar{a} \underset{1}{\square}+\bar{a} \underset{\bar{a}}{\square}\right]=Z\left(\left[X / \mathbb{Z}_{2}\right]\right),
\end{aligned}
$$

the $\mathbb{Z}_{2}=\langle\bar{a}\rangle$ orbifold of $X$, using the discrete torsion phases in [9, table D.4]. This agrees with our prediction, as expected.

\subsubsection{Summary}

We summarize the results of this analysis in table 2. As anticipated earlier, the effect of turning on discrete torsion in the $D_{4}$ orbifold is to exchange the $B(\bar{a})$ values.

\subsection{Extension of $\mathbb{Z}_{2} \times \mathbb{Z}_{4}$ to $\mathbb{Z}_{4} \rtimes \mathbb{Z}_{4}$}

Consider an orbifold $\left[X / \mathbb{Z}_{2} \times \mathbb{Z}_{4}\right]$, where the orbifold group is extended by $K=\mathbb{Z}_{2}$ to $\Gamma=\mathbb{Z}_{4} \rtimes \mathbb{Z}_{4}$, a semidirect product of two copies of $\mathbb{Z}_{4}$. We will use the same notation as in [9], and describe $\mathbb{Z}_{4} \rtimes \mathbb{Z}_{4}$ as generated by $x, y$ subject to the constraints

$$
x^{4}=1=y^{4}, \quad y=x y x .
$$

In this notation, we take $K=\left\langle x^{2}\right\rangle$, so that $K$ is central in $\mathbb{Z}_{4} \rtimes \mathbb{Z}_{4}$, and $G=\mathbb{Z}_{2} \times \mathbb{Z}_{4}$ is generated by $x K$ and $y K$.

As in the previous example, we will walk through the physical implications of each choice of $B$, to get a more complete picture of the physics here. 
In this case, the possible $B \in H^{1}\left(G, H^{1}(K, \mathrm{U}(1))\right.$ are determined by their action on the generators of $G$, as listed below:

1. $B(x K)=+1, B(y K)=+1$,

2. $B(x K)=+1, B(y K)=-1$,

3. $B(x K)=-1, B(y K)=+1$,

4. $B(x K)=-1, B(y K)=-1$.

First we will analyze these cases without discrete torsion, then we will consider the effect of adding discrete torsion $\omega \in H^{2}(\Gamma, \mathrm{U}(1))$.

\subsubsection{Without discrete torsion}

We list the predictions of our conjecture (3.2) below.

1. In the first case, $B$ is trivial, so this case reduces to ordinary decomposition $[9,16$, $35,36]$, for which

$$
\operatorname{QFT}\left(\left[X / \mathbb{Z}_{4} \rtimes \mathbb{Z}_{4}\right]\right)=\operatorname{QFT}\left(\left[X / \mathbb{Z}_{2} \times \mathbb{Z}_{4}\right] \coprod\left[X / \mathbb{Z}_{2} \times \mathbb{Z}_{4}\right]_{\text {d.t. }}\right) .
$$

2. In the second case, where $B(x K)$ is trivial but $B(y K)$ nontrivial, we have that Ker $B=\mathbb{Z}_{2} \times \mathbb{Z}_{2}=\left\langle x K, y^{2} K\right\rangle$ and Coker $B=0$, hence from (3.2) we predict that

$$
\operatorname{QFT}\left(\left[X / \mathbb{Z}_{4} \rtimes \mathbb{Z}_{4}\right]\right)=\operatorname{QFT}\left(\left[X / \mathbb{Z}_{2} \times \mathbb{Z}_{2}\right]\right) .
$$

In this case, since only the trivial irreducible representation of $K$ appears, there is no discrete torsion.

3. In the third case, where $B(x K)$ is nontrivial but $B(y K)$ trivial, we have that Ker $B=\mathbb{Z}_{4}=\langle y K\rangle$ and Coker $B=0$, hence from (3.2) we predict that

$$
\operatorname{QFT}\left(\left[X / \mathbb{Z}_{4} \rtimes \mathbb{Z}_{4}\right]\right)=\operatorname{QFT}\left(\left[X / \mathbb{Z}_{4}\right]\right) .
$$

4. In the fourth case, where both $B(x K)$ and $B(y K)$ are nontrivial, Coker $B=0$ again but Ker $B=\left(\mathbb{Z}_{2} \times \mathbb{Z}_{4}\right) / \mathbb{Z}_{2}=\mathbb{Z}_{4}=\langle x y K\rangle$, and from (3.2) we predict that

$$
\operatorname{QFT}\left(\left[X / \mathbb{Z}_{4} \rtimes \mathbb{Z}_{4}\right]\right)=\operatorname{QFT}\left(\left[X / \mathbb{Z}_{4}\right]\right) .
$$

Now, we will check the prediction in each case.

1. First, consider the trivial case $B(x K)=+1$ and $B(y K)=+1$. This corresponds to an ordinary $\mathbb{Z}_{4} \rtimes \mathbb{Z}_{4}$ orbifold, with $K=\mathbb{Z}_{2}$ acting trivially both on the underlying space as well as on the twisted sectors of $G$. This case can be described by ordinary decomposition $[9,16,35,36]$, which predicts

$$
\operatorname{QFT}\left(\left[X / \mathbb{Z}_{4} \rtimes \mathbb{Z}_{4}\right]\right)=\operatorname{QFT}\left(\left[X / \mathbb{Z}_{2} \times \mathbb{Z}_{4}\right] \coprod\left[X / \mathbb{Z}_{2} \times \mathbb{Z}_{4}\right]_{\text {d.t. }}\right),
$$


a disjoint union of two copies of $\left[X / \mathbb{Z}_{2} \times \mathbb{Z}_{4}\right]$, with discrete torsion in one copy. This can be seen directly in e.g. partition functions, as follows. Some of the $\mathbb{Z}_{2} \times \mathbb{Z}_{4}$ twisted sectors are missing from the $\mathbb{Z}_{4} \rtimes \mathbb{Z}_{4}$ orbifold partition function, such as

$$
x K \underset{y K}{\square}
$$

as the two group elements do not lift to commuting pairs in $\mathbb{Z}_{4} \rtimes \mathbb{Z}_{4}$. These are precisely the same pairs of group elements in $\mathbb{Z}_{2} \times \mathbb{Z}_{4}$ that can be weighted by nontrivial discrete torsion phases, see e.g. [9, table D.2]. Summing the two partition functions cancels out the missing twisted sectors, recovering the correct $\mathbb{Z}_{4} \rtimes \mathbb{Z}_{4}$ orbifold partition function.

2. Next, we consider the case $B(x K)=+1$ and $B(y K)=-1$. This implies that the central $K$ acts trivially on the space, but nontrivially on twisted sectors twisted by $y$, as illustrated below:

$$
x^{2} \underset{y}{\square}=-\left(1 \square_{y}^{\square}\right), \quad x^{2} \underset{y^{2}}{\square}=+\left(1 \underset{y^{2}}{\square}\right), \quad x^{2} \square_{x}=+\left(1 \square_{x}\right) .
$$

Using these relations, it is straightforward to check that in the genus-one partition function of the $\mathbb{Z}_{4} \rtimes \mathbb{Z}_{4}$ orbifold, all twisted sectors with a $y$ or $y^{3}$ cancel out, and checking multiplicities, one finds

$$
Z\left(\left[X / \mathbb{Z}_{4} \rtimes \mathbb{Z}_{4}\right]\right)=Z\left(\left[X / \mathbb{Z}_{2} \times \mathbb{Z}_{2}\right]\right),
$$

where the $\mathbb{Z}_{2} \times \mathbb{Z}_{2}=\left\langle x K, y^{2} K\right\rangle$.

3. Next, we consider the case $B(x K)=-1$ and $B(y K)=+1$. This implies that the central $K$ acts nontrivially on twisted sectors twisted by $x$, as illustrated below:

$$
x^{2} \square_{x}=-(1 \underset{x}{\square}), \quad x^{2} \underset{x^{2}}{\square}=+\left(1 \underset{x^{2}}{\square}\right), \quad x^{2} \square_{y}=+(1 \underset{y}{\square}) .
$$

As a result, twisted sector contributions with an odd number of $x$ 's along either leg cancel out from the genus-one partition function. Taking into account multiplicities, we find that the partition function is given by

$$
Z\left(\left[X / \mathbb{Z}_{4} \rtimes \mathbb{Z}_{4}\right]\right)=Z\left(\left[X / \mathbb{Z}_{4}\right]\right),
$$

where the effectively-acting $\mathbb{Z}_{4}$ on the right is $\langle y K\rangle$.

4. Next, we consider the case $B(x K)=-1$ and $B(y K)=-1$. This implies that the central $K$ acts nontrivially on twisted sectors twisted by either $x$ or $y$, as illustrated below:

$$
\begin{aligned}
& x^{2} \square_{x}=-\left(1 \square_{x}\right), \quad x^{2} \square_{x^{2}}=+\left(1 \frac{\square}{x^{2}}\right), x^{2} \square_{y}=-\left(1 \square_{y}\right) . \\
& x^{2} \square_{x y}=+\left(1 \square_{x y}\right), x^{3} \square_{x y^{3}}=+\left(x \underset{x y^{3}}{\square}\right) .
\end{aligned}
$$


As a result, genus-one twisted sectors such that the sum of the number of $x$ 's and $y$ 's on any leg are odd will cancel out. The partition function of this theory has the form

$$
\begin{aligned}
& Z\left(\left[X / \mathbb{Z}_{4} \rtimes \mathbb{Z}_{4}\right]\right) \\
& =\frac{1}{\left|\mathbb{Z}_{4} \rtimes \mathbb{Z}_{4}\right|} \sum_{g h=h g} g \square, \\
& =\frac{4}{\left|\mathbb{Z}_{4} \rtimes \mathbb{Z}_{4}\right|}\left[1 \underset{1}{\square}+1 \underset{y^{2} K}{\square}+1 \underset{x y K}{\square}+1 \square_{x y^{3} K}+x y K \square_{1}+x y K \underset{x y K}{\square}+x y K \underset{y^{2} K}{\square}\right. \\
& +x y K \underset{x y^{3} K}{\square}+y^{2} K \underset{1}{\square}+y^{2} K \underset{y^{2} K}{\square}+y^{2} K \underset{x y K}{\square}+y^{2} K \underset{x y^{3} K}{\square} \\
& \left.+x y^{3} K \square_{1}+x y^{3} K \underset{x y K}{\square}+x y^{3} K \underset{x y^{3} K}{\square}+x y^{3} K \underset{y^{2} K}{\square}\right], \\
& =Z\left(\left[X / \mathbb{Z}_{4}\right]\right),
\end{aligned}
$$

where the $\mathbb{Z}_{4}=\langle x y K\rangle$, using the fact that $(x y)^{2}=y^{2},(x y)^{3}=x y^{3}$ in $\mathbb{Z}_{4} \rtimes \mathbb{Z}_{4}$.

As expected, each case matches our prediction (3.2).

\subsubsection{With discrete torsion}

Now, let us turn on discrete torsion in the $\mathbb{Z}_{4} \rtimes \mathbb{Z}_{4}$ orbifold. Since $H^{2}\left(\mathbb{Z}_{4} \rtimes \mathbb{Z}_{4}, \mathrm{U}(1)\right)=\mathbb{Z}_{2}$, there is exactly one nontrivial choice.

Before describing the physics results in this case, let us take a moment to analyze this case formally and predict the result. In the notation of [9], this is a case in which $\iota^{*} \omega=0$ and $\beta(\omega) \neq 0$, where $\beta(\omega)$ is the image of $\omega \in H^{2}(\Gamma, \mathrm{U}(1))$ in $H^{1}\left(G, H^{1}(K, \mathrm{U}(1))\right)$. As computed in $[9$, section 5.6],

$$
\beta(\omega)\left(x K, x^{2}\right)=+1, \quad \beta(\omega)\left(y K, x^{2}\right)=-1,
$$

so the effect of turning on $\omega$ should be to flip the value of $B(y K)$.

Now, let us check this prediction, by walking through each case explicitly.

1. In the first case, $B$ itself is trivial. Now, the $\Gamma=\mathbb{Z}_{4} \rtimes \mathbb{Z}_{4}$ orbifold with trivially-acting central $K=\mathbb{Z}_{2}=\left\langle x^{2}\right\rangle$ and discrete torsion was discussed in detail in [9, section 5.6], where it was argued that

$$
\operatorname{QFT}\left([X / \Gamma]_{\text {d.t. }}\right)=\operatorname{QFT}\left(\left[X / \mathbb{Z}_{2} \times \mathbb{Z}_{2}\right]\right) \text {. }
$$

2. Next, we consider the case $B(x K)=+1, B(y K)=-1$, with discrete torsion in the $\Gamma=\mathbb{Z}_{4} \rtimes \mathbb{Z}_{4}$ orbifold. As observed in the case without discrete torsion, $B$ encodes an action of $K$ on the twisted sectors twisted by $y$, as described in equation (4.108). In particular, $B$ relates by a sign twisted sectors with an odd number of powers of $y$. The twisted sector phases of $\mathbb{Z}_{4} \rtimes \mathbb{Z}_{4}$, as listed in [9, table D.6], have the same effect: twisted sectors with odd powers of $y$ are also related by signs. The combined effect of both $B$ and the discrete torsion is, therefore, to cancel out, leaving us with a 
$\Gamma=\mathbb{Z}_{4} \rtimes \mathbb{Z}_{4}$ orbifold in which $K=\left\langle x^{2}\right\rangle$ acts trivially on both the underlying space $X$ as well as twisted sectors. This can be described by ordinary decomposition, and as we described earlier, and can be checked directly in partition functions, we have

$$
\operatorname{QFT}\left(\left[X / \mathbb{Z}_{4} \rtimes \mathbb{Z}_{4}\right]\right)=\operatorname{QFT}\left(\left[X / \mathbb{Z}_{2} \times \mathbb{Z}_{4}\right] \coprod\left[X / \mathbb{Z}_{2} \times \mathbb{Z}_{4}\right]_{\text {d.t. }}\right),
$$

a disjoint union of two $\mathbb{Z}_{2} \times \mathbb{Z}_{4}$ orbifolds.

3. Next, we consider the case $B(x K)=-1, B(y K)=+1$. In this case, as before, roughly, the $B$ by itself would cancel out genus-one twisted sectors with odd powers of $x$, and the discrete torsion would cancel out genus-one twisted sectors with odd powers of $y$; however, sectors involving e.g. $x y, x y^{3}$ survive. Using [9, table D.6], the genus-one partition function is

$$
\begin{aligned}
& Z\left(\left[X / \mathbb{Z}_{4} \rtimes \mathbb{Z}_{4}\right]_{\text {d.t. }}\right) \\
& =\frac{1}{\left|\mathbb{Z}_{4} \rtimes \mathbb{Z}_{4}\right|} \sum_{g h=h g} \epsilon(g, h)(g \square), \\
& =\frac{4}{\left|\mathbb{Z}_{4} \rtimes \mathbb{Z}_{4}\right|}\left[1 \square+1 \underset{y^{2} K}{\square}+1 \underset{x y K}{\square}+1 \square_{x y^{3} K}+x y K \square_{1}+x y K \underset{x y K}{\square}+x y K \underset{y^{2} K}{\square}\right. \\
& +x y K \underset{x y^{3} K}{\square}+y^{2} K \underset{1}{\square}+y^{2} K \underset{y^{2} K}{\square}+y^{2} K \underset{x y K}{\square}+y^{2} K \underset{x y^{3} K}{\square} \\
& \left.+x y^{3} K \underset{1}{\square}+x y^{3} K \underset{x y K}{\square}+x y^{3} K \underset{x y^{3} K}{\square}+x y^{3} K \underset{y^{2} K}{\square}\right] \text {, } \\
& =Z\left(\left[X / \mathbb{Z}_{4}\right]\right),
\end{aligned}
$$

where the $\mathbb{Z}_{4}=\langle x y K\rangle$, using the fact that $(x y)^{2}=y^{2},(x y)^{3}=x y^{3}$ in $\mathbb{Z}_{4} \rtimes \mathbb{Z}_{4}$.

4. Finally, we consider the case $B(x K)=-1$ and $B(y K)=-1$, for a $\mathbb{Z}_{4} \rtimes \mathbb{Z}_{4}$ orbifold with discrete torsion. Using the methods above, it is straightforward to compute that

$$
\begin{aligned}
& Z\left(\left[X / \mathbb{Z}_{4} \rtimes \mathbb{Z}_{4}\right]_{\text {d.t. }}\right) \\
& =\frac{1}{\left|\mathbb{Z}_{4} \rtimes \mathbb{Z}_{4}\right|} \sum_{g h=h g} \epsilon(g, h)(g \square), \\
& =\frac{4}{\left|\mathbb{Z}_{4} \rtimes \mathbb{Z}_{4}\right|}\left[1 \underset{1}{\square}+1 \underset{y K}{\square}+1 \underset{y^{2} K}{\square}+1 \underset{y^{3} K}{\square}+y K \underset{1}{\square}+y K \underset{y K}{\square}+y K \underset{y^{2} K}{\square}+y K \underset{y^{3} K}{\square}\right. \\
& +y^{2} K \underset{1}{\square}+y^{2} K \underset{y K}{\square}+y^{2} K \underset{y^{2} K}{\square}+y^{2} K \underset{y^{3} K}{\square}+y^{3} K \underset{1}{\square}+y^{3} K \underset{y K}{\square} \\
& \left.+y^{3} K \underset{y^{2} K}{\square}+y^{3} K \underset{y^{3} K}{\square}\right] \\
& =Z\left(\left[X / \mathbb{Z}_{4}\right]\right),
\end{aligned}
$$

where the $\mathbb{Z}_{4}=\langle y K\rangle$. 


\begin{tabular}{c|cc|cc}
\hline Case & $B(x K)$ & $B(y K)$ & Without discrete torsion & With discrete torsion \\
\hline 1 & +1 & +1 & {$\left[X / \mathbb{Z}_{2} \times \mathbb{Z}_{4}\right] \amalg\left[X / \mathbb{Z}_{2} \times \mathbb{Z}_{4}\right]_{\text {d.t. }}$} & {$\left[X / \mathbb{Z}_{2} \times \mathbb{Z}_{2}\right]$} \\
2 & +1 & -1 & {$\left[X / \mathbb{Z}_{2} \times \mathbb{Z}_{2}=\left\langle x K, y^{2} K\right\rangle\right]$} & {$\left[X / \mathbb{Z}_{2} \times \mathbb{Z}_{4}\right] \amalg\left[X / \mathbb{Z}_{2} \times \mathbb{Z}_{4}\right]_{\text {d.t. }}$} \\
3 & -1 & +1 & {$\left[X / \mathbb{Z}_{4}=\langle y K\rangle\right]$} & {$\left[X / \mathbb{Z}_{4}=\langle x y K\rangle\right]$} \\
4 & -1 & -1 & {$\left[X / \mathbb{Z}_{4}=\langle x y K\rangle\right]$} & {$\left[X / \mathbb{Z}_{4}=\langle y K\rangle\right]$} \\
\hline
\end{tabular}

Table 3. Summary of results for $D_{4}$ orbifold extending $\mathbb{Z}_{2} \times \mathbb{Z}_{2}$ orbifold.

\subsubsection{Summary}

These results are summarized in table 3 . As anticipated, the effect of discrete torsion is to flip the value of $\beta(y K)$.

\subsection{Extension of $D_{4}$ to $\mathbb{Z}_{4} \rtimes \mathbb{Z}_{4}$}

In this section, we will discuss an example of a $D_{4}$ orbifold being extended by $K=\mathbb{Z}_{2}$ to $\Gamma=\mathbb{Z}_{4} \rtimes \mathbb{Z}_{4}$. In this example, the extension is the central subgroup $K=\left\langle y^{2}\right\rangle$, in the notation of $[9]$.

We will follow the same notation as [9], so we denote the elements of $D_{4}$ by

$$
\{1, z, a, b, a z, b z, a b, b a=a b z\}
$$

where

$$
a^{2}=1=b^{4}, \quad b^{2}=z,
$$

with $z$ central in $D_{4}$. The projection $\pi: \Gamma \rightarrow D_{4}$ maps

$$
\pi(x)=b=\pi\left(x y^{2}\right), \quad \pi(y)=a=\pi\left(y^{3}\right) .
$$

The possible $B \in H^{1}\left(G, H^{1}(K, \mathrm{U}(1))\right.$ are enumerated by their valued on the generators below:

1. $B(a)=+1, B(b)=+1$,

2. $B(a)=+1, B(b)=-1$,

3. $B(a)=-1, B(b)=+1$,

4. $B(a)=-1, B(b)=-1$.

We will first consider cases without discrete torsion, then cases with discrete torsion $\omega \in H^{2}\left(\mathbb{Z}_{4} \rtimes \mathbb{Z}_{4}, \mathrm{U}(1)\right)$.

\subsubsection{Without discrete torsion}

From section 3, we predict that

$$
\operatorname{QFT}\left([X / \Gamma]_{B}\right)=\operatorname{QFT}\left(\left[\frac{X \times \widehat{\operatorname{Coker} B}}{\operatorname{Ker} B}\right]_{\hat{\omega}_{0}}\right),
$$


where $\hat{\omega}_{0}$ is the discrete torsion that one computes from ordinary decomposition, without a quantum symmetry. Here, since $K$ is central, $\hat{\omega}_{0}$ is the image of the extension class under the corresponding irreducible representation. We studied this example without a quantum symmetry in $[9$, section 6.2$]$, where we argued that $\hat{\omega}_{0}=0$, so to predict each case, one merely needs to compute the kernel and cokernel of $B$, which we do below.

1. First, consider the case $B(a)=+1, B(b)=+1$, meaning the case without a quantum symmetry. Since $B$ is trivial, the kernel is $G=D_{4}$, and the cokernel is $\hat{\mathbb{Z}}_{2}$, so the result reduces to that of ordinary decomposition:

$$
\operatorname{QFT}\left([X / \Gamma]_{B}\right)=\operatorname{QFT}\left(\coprod_{2}\left[X / D_{4}\right]\right) \text {. }
$$

2. Next, consider the case $B(a)=+1, B(b)=-1$. In this case, the cokernel of $B$ is trivial, but

$$
\operatorname{Ker} B=\left\langle a, b^{2}\right\rangle=\left\langle y K, x^{2} K\right\rangle=\mathbb{Z}_{2} \times \mathbb{Z}_{2},
$$

hence we predict

$$
\operatorname{QFT}\left([X / \Gamma]_{B}\right)=\operatorname{QFT}\left(\left[X / \mathbb{Z}_{2} \times \mathbb{Z}_{2}\right]\right)
$$

3. Next, consider the case $B(a)=-1, B(b)=+1$. In this case, the cokernel of $B$ is trivial, but

$$
\operatorname{Ker} B=\left\langle a^{2}, b\right\rangle=\langle b\rangle=\langle x K\rangle=\mathbb{Z}_{4},
$$

hence we predict

$$
\operatorname{QFT}\left([X / \Gamma]_{B}\right)=\operatorname{QFT}\left(\left[X / \mathbb{Z}_{4}\right]\right) .
$$

4. Next, consider the case $B(a)=-1, B(b)=-1$. In this case, the cokernel of $B$ is trivial, but

$$
\text { Ker } B=\left\langle a^{2}, a b, b^{2}\right\rangle=\left\langle a b, b^{2}\right\rangle=\left\langle x y K, y^{2} K\right\rangle=\mathbb{Z}_{2} \times \mathbb{Z}_{2},
$$

hence we predict

$$
\operatorname{QFT}\left([X / \Gamma]_{B}\right)=\operatorname{QFT}\left(\left[X / \mathbb{Z}_{2} \times \mathbb{Z}_{2}\right]\right) .
$$

Next, we briefly review the physics each case for $B$, without discrete torsion. In each case, we find that partition function computations verify the predictions above. (We have included the case of trivial $B$ for completeness.)

1. First, consider the case that $B(a)=+1$ and $B(b)=+1$. This is an ordinary $\mathbb{Z}_{4} \rtimes \mathbb{Z}_{4}$ orbifold, with $K=\mathbb{Z}_{2}$ acting trivially both on the underlying space as well as on the twisted sectors of $G$. This case can be described by ordinary decomposition $[9,16,35,36]$, which predicts (see $[9$, section 6.2$]$ )

$$
\operatorname{QFT}\left(\left[X / \mathbb{Z}_{4} \rtimes \mathbb{Z}_{4}\right]\right)=\operatorname{QFT}\left(\coprod_{2}\left[X / D_{4}\right]\right),
$$

or in other words, that this theory is equivalent to a disjoint union of two $D_{4}$ orbifolds, neither with discrete torsion. 
2. Next, we consider the case that $B(a)=+1$ and $B(b)=-1$. This implies that the central $K$ acts trivially on the space, but nontrivially on twisted sectors twisted by $x$, for example

$$
y^{2} \underset{x}{\square_{x}}=-(1 \underset{x}{\square}), \quad x y^{2} \underset{y}{\square_{y}}=+(x \underset{y}{\square}), \quad y^{2} \underset{y^{2}}{\square}=+\left(1 \square_{y^{2}}\right)=+\left(1 \square_{1}^{\square}\right) .
$$

Essentially as a result, in the genus-one partition function, twisted sectors with an odd number of powers of $x$ on either leg cancel out. It is straightforward to compute that the genus-one partition function is

$$
\begin{aligned}
Z\left(\left[X / \mathbb{Z}_{4} \rtimes \mathbb{Z}_{4}\right]\right) & =\frac{1}{\left|\mathbb{Z}_{4} \rtimes \mathbb{Z}_{4}\right|} \sum_{g h=h g} g \bigsqcup_{h}, \\
& =Z\left(\left[X / \mathbb{Z}_{2} \times \mathbb{Z}_{2}\right]\right),
\end{aligned}
$$

an orbifold by $\mathbb{Z}_{2} \times \mathbb{Z}_{2}=\left\langle x^{2} K, y K\right\rangle$.

3. Next, we consider the case that $B(a)=-1$ and $B(b)=+1$. This implies that the central $K$ acts trivially on the space but nontrivially on twisted sectors twisted by $y$, for example

$$
y^{2} \square_{y}=-\left(1 \square_{y}\right), \quad y^{2} \square_{x}=+\left(1 \square_{x}\right) .
$$

Essentially as a result, in the genus-one partition function, twisted sectors with an odd number of powers of $y$ on either leg cancel out. It is straightforward to compute that the genus-one partition function is

$$
\begin{aligned}
Z\left(\left[X / \mathbb{Z}_{4} \rtimes \mathbb{Z}_{4}\right]\right) & =\frac{1}{\left|\mathbb{Z}_{4} \rtimes \mathbb{Z}_{4}\right|} \sum_{g h=h g} g \square, \\
& =Z\left(\left[X / \mathbb{Z}_{4}\right]\right),
\end{aligned}
$$

an orbifold by $\mathbb{Z}_{4}=\langle x K\rangle$.

4. Finally, we consider the case that $B(a)=-1$ and $B(b)=-1$. This implies that the central $K$ acts trivially on the space but nontrivially on twisted sectors twisted by either $a$ or $b$, for example

$$
y^{2} \square_{x}=-\left(1 \square_{x}\right), \quad y^{2} \square_{y}=-\left(1 \square_{y}\right), \quad y^{2} \square_{x y}=+\left(1 \square_{x y}\right) .
$$

Essentially as a result, in the genus-one partition function, twisted sector with an odd number of powers of $x$ and $y$ on either leg cancel out. It is straightforward to compute that the genus-one partition function is

$$
\begin{aligned}
Z\left(\left[X / \mathbb{Z}_{4} \rtimes \mathbb{Z}_{4}\right]\right) & =\frac{1}{\left|\mathbb{Z}_{4} \rtimes \mathbb{Z}_{4}\right|} \sum_{g h=h g} g \square, \\
& =Z\left(\left[X / \mathbb{Z}_{2} \times \mathbb{Z}_{2}\right]\right),
\end{aligned}
$$

an orbifold by $\mathbb{Z}_{2} \times \mathbb{Z}_{2}=\left\langle x^{2} K, x y K\right\rangle$, without discrete torsion. 


\subsubsection{With discrete torsion}

Now, let us turn on discrete torsion $\omega$ in the $\mathbb{Z}_{4} \rtimes \mathbb{Z}_{4}$ orbifold. As discussed in [9, section 6.2], $\iota^{*} \omega=0, \beta(\omega)=0$, and in fact this discrete torsion is a pullback from $\bar{\omega} \in H^{2}\left(D_{4}, \mathrm{U}(1)\right)$.

From section 3 , we predict

$$
\operatorname{QFT}\left([X / \Gamma]_{B, \omega}\right)=\operatorname{QFT}\left(\left[\frac{X \times \widehat{\operatorname{Coker} B}}{\operatorname{Ker} B}\right]_{\bar{\omega}+\hat{\omega}_{0}}\right) .
$$

From our discussion in the case without discrete torsion, we know that $\hat{\omega}_{0}=0$, and we have already computed the kernels and cokernels, so we merely need to add the restriction of $\bar{\omega}$ to the kernel in each case to get our prediction, as we list below. We include the case that $B$ is trivial for completeness.

1. First, consider the case that $B$ is trivial: $B(a)=+1, B(b)=+1$. In this case, the kernel is $D_{4}$ and the cokernel is $\hat{\mathbb{Z}}_{2}$, so we find

$$
\operatorname{QFT}\left([X / \Gamma]_{B, \omega}\right)=\operatorname{QFT}\left(\coprod_{2}\left[X / D_{4}\right]_{\bar{\omega}}\right)
$$

as discussed in [9, section 6.2].

2. Next, consider the case that $B(a)=+1, B(b)=-1$. Here, the cokernel is trivial and the kernel is $\left\langle y K, x^{2} K\right\rangle=\mathbb{Z}_{2} \times \mathbb{Z}_{2}$, so we predict

$$
\operatorname{QFT}\left([X / \Gamma]_{B, \omega}\right)=\operatorname{QFT}\left(\left[X / \mathbb{Z}_{2} \times \mathbb{Z}_{2}\right]_{\bar{\omega}}\right) .
$$

3. Next, consider the case that $B(a)=-1, B(b)=+1$. Here, the cokernel is trivial and the kernel is $\langle x K\rangle=\mathbb{Z}_{4}$. Since this group has no discrete torsion, the restriction of $\bar{\omega}$ is trivial, so we predict

$$
\operatorname{QFT}\left([X / \Gamma]_{B, \omega}\right)=\operatorname{QFT}\left(\left[X / \mathbb{Z}_{4}\right]\right) .
$$

4. Next, consider the case that $B(a)=-1, B(b)=-1$. Here, the cokernel is trivial and the kernel is $\left\langle x y K, y^{2} K\right\rangle=\mathbb{Z}_{2} \times \mathbb{Z}_{2}$, so we predict

$$
\operatorname{QFT}\left([X / \Gamma]_{B, \omega}\right)=\operatorname{QFT}\left(\left[X / \mathbb{Z}_{2} \times \mathbb{Z}_{2}\right]_{\bar{\omega}}\right) .
$$

Next, we compute genus-one partition functions. In each case, we find that the partition function computations are consistent with the predictions above.

1. First, consider the case that $B(a)=+1$ and $B(b)=+1$. This is an ordinary $\mathbb{Z}_{4} \rtimes \mathbb{Z}_{4}$ orbifold with discrete torsion, with trivially-acting $K=\mathbb{Z}_{2}=\left\langle y^{2}\right\rangle$. This particular example was discussed in [9, section 6.2], where it was argued

$$
\operatorname{QFT}\left(\left[X / \mathbb{Z}_{4} \rtimes \mathbb{Z}_{4}\right]_{\text {d.t. }}\right)=\operatorname{QFT}\left(\coprod_{2}\left[X / D_{4}\right]_{\bar{\omega}}\right),
$$

or in other words that this theory is equivalent to a disjoint union of two $D_{4}$ orbifolds, each with discrete torsion $\bar{\omega}$. 
2. Next, we consider the case that $B(a)=+1$ and $B(b)=-1$, and turn on discrete torsion in the $\mathbb{Z}_{4} \rtimes \mathbb{Z}_{4}$ orbifold. The analysis proceeds very similarly to the case we considered previously without discrete torsion, as the cancellations due to $\beta$ involve pairs which have the same discrete torsion phases, using the phases in [9, table D.6]. It is straightforward to check that the genus-one partition function is

$$
\begin{aligned}
Z\left(\left[X / \mathbb{Z}_{4} \rtimes \mathbb{Z}_{4}\right]_{\text {d.t. }}\right) & =\frac{1}{\left|\mathbb{Z}_{4} \rtimes \mathbb{Z}_{4}\right|} \sum_{g h=h g} \epsilon(g, h)(g \square), \\
& =Z\left(\left[X / \mathbb{Z}_{2} \times \mathbb{Z}_{2}\right]_{\text {d.t. }}\right),
\end{aligned}
$$

an orbifold by $\mathbb{Z}_{2} \times \mathbb{Z}_{2}=\left\langle x^{2} K, y K\right\rangle$ with discrete torsion.

3. Next, we consider the case that $B(a)=-1$ and $B(b)=+1$, and turn on discrete torsion in the $\mathbb{Z}_{4} \rtimes \mathbb{Z}_{4}$ orbifold. The analysis proceeds very similarly to the case we considered previously without discrete torsion, as the cancellations due to $\beta$ involve pairs which have the same discrete torsion phases, using the phases in [9, table D.6]. It is straightforward to check that the genus-one partition function is

$$
\begin{aligned}
Z\left(\left[X / \mathbb{Z}_{4} \rtimes \mathbb{Z}_{4}\right]_{\text {d.t. }}\right) & =\frac{1}{\left|\mathbb{Z}_{4} \rtimes \mathbb{Z}_{4}\right|} \sum_{g h=h g} \epsilon(g, h)(g \square), \\
& =Z\left(\left[X / \mathbb{Z}_{4}\right]\right),
\end{aligned}
$$

an orbifold by $\mathbb{Z}_{4}=\langle x K\rangle$.

4. Finally, we consider the case that $B(a)=-1$ and $B(b)=-1$, and turn on discrete torsion in the $\mathbb{Z}_{4} \rtimes \mathbb{Z}_{4}$ orbifold. The analysis proceeds very similarly to the case we considered previously without discrete torsion, as the cancellations due to $\beta$ involve pairs which have the same discrete torsion phases, using the phases in [9, table D.6]. It is straightforward to check that the genus-one partition function is

$$
\begin{aligned}
Z\left(\left[X / \mathbb{Z}_{4} \rtimes \mathbb{Z}_{4}\right]_{\text {d.t. }}\right) & =\frac{1}{\left|\mathbb{Z}_{4} \rtimes \mathbb{Z}_{4}\right|} \sum_{g h=h g} \epsilon(g, h)(g \square), \\
& =Z\left(\left[X / \mathbb{Z}_{2} \times \mathbb{Z}_{2}\right]_{\text {d.t. }}\right),
\end{aligned}
$$

an orbifold by $\mathbb{Z}_{2} \times \mathbb{Z}_{2}=\left\langle x^{2} K, x y K\right\rangle$, with discrete torsion.

\subsubsection{Summary}

We summarize the results of this analysis in table 4 .

\subsection{Extension of $\mathbb{Z}_{2} \times \mathbb{Z}_{2}$ to $D_{4} \times \mathbb{Z}_{2} \times \mathbb{Z}_{2}$}

In this section, we begin with a $G=\mathbb{Z}_{2} \times \mathbb{Z}_{2}=\langle\bar{a}, \bar{b}\rangle$ orbifold, which we centrally extend by $K=\left(\mathbb{Z}_{2}\right)^{3}$ to $\Gamma=D_{4} \times \mathbb{Z}_{2} \times \mathbb{Z}_{2}$, where we write $\left(\mathbb{Z}_{2}\right)^{2}=\langle x, y\rangle$. In particular, in this example, the restriction of discrete torsion in $\Gamma$ to $K$ can be nontrivial, so we will be able to explore cases in which $\iota^{*} \omega \neq 0$. 


\begin{tabular}{c|cc|cc}
\hline Case & $B(a)$ & $B(b)$ & Without discrete torsion & With discrete torsion \\
\hline 1 & +1 & +1 & {$\left[X / D_{4}\right] \amalg\left[X / D_{4}\right]$} & {$\left[X / D_{4}\right]_{\bar{\omega}} \amalg\left[X / D_{4}\right]_{\bar{\omega}}$} \\
2 & +1 & -1 & {$\left[X / \mathbb{Z}_{2} \times \mathbb{Z}_{2}=\left\langle x^{2} K, y K\right\rangle\right]$} & {$\left[X / \mathbb{Z}_{2} \times \mathbb{Z}_{2}=\left\langle x^{2} K, y K\right\rangle\right]_{\text {d.t. }}$} \\
3 & -1 & +1 & {$\left[X / \mathbb{Z}_{4}=\langle x K\rangle\right]$} & {$\left[X / \mathbb{Z}_{4}=\langle x K\rangle\right]$} \\
4 & -1 & -1 & {$\left[X / \mathbb{Z}_{2} \times \mathbb{Z}_{2}=\left\langle x^{2} K, x y K\right\rangle\right]$} & {$\left[X / \mathbb{Z}_{2} \times \mathbb{Z}_{2}=\left\langle x^{2} K, x y K\right\rangle\right]_{\text {d.t. }}$} \\
\hline
\end{tabular}

Table 4. Summary of results for $\mathbb{Z}_{4} \rtimes \mathbb{Z}_{4}$ orbifold extending $D_{4}$ orbifold.

\subsubsection{Without discrete torsion}

First, let us assume that the $\Gamma$ orbifold has no discrete torsion, and work out the consequences. We will describe details for some representative values of $B \in H^{1}\left(G, H^{1}(K, \mathrm{U}(1))\right)$ below.

First, if $B$ is trivial, then the $[X / \Gamma]$ orbifold is described by decomposition $[9,16,35,36]$, which predicts

$$
\operatorname{QFT}([X / \Gamma])=\operatorname{QFT}\left(\coprod_{4}\left[X / D_{4}\right]\right),
$$

and furthermore (see esp. [16, section 5.2]),

$$
\operatorname{QFT}\left(\left[X / D_{4}\right]\right)=\operatorname{QFT}\left(\left[X / \mathbb{Z}_{2} \times \mathbb{Z}_{2}\right] \coprod\left[X / \mathbb{Z}_{2} \times \mathbb{Z}_{2}\right]_{\text {d.t. }}\right),
$$

so in particular,

$$
\operatorname{QFT}([X / \Gamma])=\operatorname{QFT}\left(\coprod_{4}\left[X / \mathbb{Z}_{2} \times \mathbb{Z}_{2}\right] \coprod_{4}\left[X / \mathbb{Z}_{2} \times \mathbb{Z}_{2}\right]_{\text {d.t. }}\right) .
$$

It is straightforward to check using by-now standard methods that closed-string partition functions agree with the predictions above, so we move on to less trivial cases.

Next, consider the case that $B(\bar{a})=(+,-,+)$ but $B(\bar{b})$ is trivial. The notation means that $z \in D_{4}$ and $y$ act trivially on $\bar{a}$, but $x$ acts nontrivially. In this case, $\operatorname{Ker} B=\mathbb{Z}_{2}=\langle\bar{b}\rangle$, and Coker $B=\left(\mathbb{Z}_{2}\right)^{2}$, so we predict

$$
\operatorname{QFT}\left([X / \Gamma]_{B}\right)=\operatorname{QFT}\left(\left[\frac{X \times \widehat{\mathbb{Z}}_{2 \times \mathbb{Z}_{2}}}{\mathbb{Z}_{2}}\right]\right)=\operatorname{QFT}\left(\coprod_{4}\left[X / \mathbb{Z}_{2}=\langle\bar{b}\rangle\right]\right) .
$$

The same kernel and cokernel arise, and so one has the same prediction, for the cases $\beta(\bar{a})=(+,+,-)$ and $(+,-,-)$ while $\beta(\bar{b})=(+,+,+)$.

It is straightforward to verify this prediction using partition functions. Returning to the case $\beta(\bar{a})=(+,-,+)$, in the partition function

$$
Z\left([X / \Gamma]_{B}\right)=\frac{1}{|\Gamma|} \sum_{g h=h g} g \square
$$

it is straightforward to check that all contributions from genus-one twisted sectors involving $a$ are cancelled out by corresponding sectors with $x$ 's. The remaining genus-one twisted 
sectors project to those of a $\langle\bar{b}\rangle=\mathbb{Z}_{2}$ orbifold, with multiplicity $4^{3}$ (arising from possible multiplications by $z, x$, and $y$.) As a result,

$$
Z\left([X / \Gamma]_{B}\right)=\frac{4^{3}}{|\Gamma|}(\langle\bar{b}\rangle \text { twisted sectors })=Z\left(\coprod_{4}\left[X / \mathbb{Z}_{2}=\langle\bar{b}\rangle\right]\right)
$$

confirming the prediction above. The other cases above are treated identically.

Certainly a variety of other cases exist, but as we feel we have sufficient examples of this form, for the sake of brevity we will move on.

\subsubsection{With discrete torsion in $\mathbb{Z}_{2} \times \mathbb{Z}_{2} \subset \Gamma$}

Next, let us assume that there is discrete torsion in $\mathbb{Z}_{2} \times \mathbb{Z}_{2} \subset \Gamma$. Note that in this case, $\iota^{*} \omega \neq 0$. As a result, we do not have a prediction for decomposition here, but we can collect results experimentally.

If $B$ is trivial, then this reduces to an example studied in [9, section 4.5], where it was shown

$$
\operatorname{QFT}\left([X / \Gamma]_{\omega}\right)=\operatorname{QFT}\left(\left[X / D_{4}\right]\right)=\operatorname{QFT}\left(\left[X / \mathbb{Z}_{2} \times \mathbb{Z}_{2}\right] \coprod\left[X / \mathbb{Z}_{2} \times \mathbb{Z}_{2}\right]_{\text {d.t. }}\right) .
$$

Suppose next that $B(\bar{a})=(+,-,+)$ and $B(\bar{b})$ trivial. We will see that the genus-one partition function is the same as that of $\left[X / D_{4}\right]$.

First, consider the genus-one twisted sectors that project to

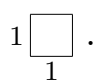

Excluding contributions from $z$ (the generator of the center of $D_{4}$ ), the contributing sectors are

$$
\begin{aligned}
& 1 \square_{1}+x \square_{1}+y \square_{1}+x y \square_{1}+1 \square_{x}+1 \square_{y}+1 \square_{x y}+x \square_{x}-x \underset{y}{\square_{y}}-x \square_{x y}-y \square_{x} \\
& +y \square_{y}-y \square_{x y}-x y \square_{x}-x y \square_{y}+x y \square_{x y},
\end{aligned}
$$

where the signs are entirely due to discrete torsion. Since none of the group elements project to elements of $G$ involving $\bar{a}$ or products thereof, $B$ does not contribute any relative signs. Thus, we see that, ignoring contributions from $z$ 's, these genus one sectors are equivalent to

$$
(10-6)(1 \underset{1}{1 \square})=(4)(1 \underset{1}{1 \square}) \text {. }
$$

Now, consider the genus-one twisted sectors that project to

$$
\bar{a} \square .
$$

Excluding contributions from $z$ (the generator of the center of $D_{4}$ ), the contributing sectors are

$$
\begin{aligned}
& a \square_{1}+x a \square_{1}+y a \square_{1}+x y a \square_{1}+a \square_{x}+a \square_{y}+a \square_{x y}+x a \square_{x}-x a \square_{y}-x a \square_{x y}-y a \square_{x} \\
& +y a \square_{y}-y a \square_{x y}-x y a \square_{x}-x y a \square_{y}+x y a \square_{x y}
\end{aligned}
$$


where the signs listed are entirely due to discrete torsion. In this case, $B$ contributes nontrivial relative phases. For example,

$$
a \square_{x}=-(a \square)
$$

Taking into account these signs, as well as discrete torsion, we find that the sum of the genus-one twisted sectors above is again

$$
\text { (4) }(\bar{a} \underset{\square}{\square}) \text {. }
$$

Other genus-one sectors involving factors of $a$ are similar.

Putting these contributions together, and taking into account contributions from $z$ 's, we find

$$
\begin{aligned}
Z\left([X / \Gamma]_{B, \omega}\right) & =\frac{(4)}{\left|D_{4} \times\left(\mathbb{Z}_{2}\right)^{2}\right|}\left(\text { sectors of } D_{4} \text { orbifold }\right) \\
& =Z\left(\left[X / D_{4}\right]\right)=Z\left(\left[X / \mathbb{Z}_{2} \times \mathbb{Z}_{2}\right] \coprod\left[X / \mathbb{Z}_{2} \times \mathbb{Z}_{2}\right]_{\text {d.t. }}\right) .
\end{aligned}
$$

\subsubsection{With discrete torsion in $D_{4} \subset \Gamma$}

Finally, let us consider the possibility that there is discrete torsion in $D_{4} \subset \Gamma$. In these cases, $\iota^{*} \omega=0$ but $\beta(\omega) \neq 0$.

If $B$ is trivial, then from decomposition, this reduces to $\left|\mathbb{Z}_{2} \times \mathbb{Z}_{2}\right|=4$ copies of $\left[X / D_{4}\right]_{\omega}$, for which it was shown in $[9$, section 5.5$]$ that

$$
\operatorname{QFT}\left(\left[X / D_{4}\right]_{\omega}\right)=\operatorname{QFT}\left(\left[X / \mathbb{Z}_{2}=\langle\bar{b}\rangle\right]\right)
$$

hence

$$
\operatorname{QFT}\left([X / \Gamma]_{\omega}\right)=\operatorname{QFT}\left(\coprod_{4}\left[X / D_{4}\right]_{\omega}\right)=\operatorname{QFT}\left(\coprod_{4}\left[X / \mathbb{Z}_{2}=\langle\bar{b}\rangle\right]\right) .
$$

Suppose instead that $B(\bar{a})=(+,-,+)$ and $B(\bar{b})$ is trivial. Then from section 3 , we predict that

$$
\operatorname{QFT}\left([X / \Gamma]_{B, \omega}\right)=\operatorname{QFT}\left(\left[\frac{X \times \operatorname{Coker~} B / \beta(\omega)}{\operatorname{Ker} B / \beta(\omega)}\right]\right),
$$

where $\beta(\omega)$ was computed in the closely-related case of a $D_{4}$ orbifold with discrete torsion and trivially-acting center in [9, section 5.5]. In the notation of this section,

$$
\beta(\omega)(\bar{a})=(-,+,+), \quad \beta(\omega)(\bar{b})=(+,+,+),
$$

so that

$$
(B / \beta(\omega))(\bar{a})=(-,-,+), \quad(B / \beta(\omega))(\bar{b})=(+,+,+) .
$$

From this we compute

$$
\operatorname{Ker} B / \beta(\omega)=\mathbb{Z}_{2}=\langle\bar{b}\rangle, \quad \text { Coker } B / \beta(\omega)=\mathbb{Z}_{2} \times \mathbb{Z}_{2} .
$$




\begin{tabular}{c|c|c|c|c}
\hline$B(\bar{a})$ & $B(\bar{b})$ & $\mathrm{W} /$ o d.t. & D.t. in $\left(\mathbb{Z}_{2}\right)^{2}$ & D.t. in $D_{4}$ \\
\hline$(+++)$ & $(+++)$ & $\coprod_{4}\left[X / D_{4}\right]$ & {$\left[X / D_{4}\right]$} & $\coprod_{4}\left[X / \mathbb{Z}_{2}=\langle\bar{b}\rangle\right]$ \\
\hline $\begin{array}{c}(+ \pm \mp) \\
(+--)\end{array}$ & $(+++)$ & $\amalg_{4}\left[X / \mathbb{Z}_{2}=\langle\bar{b}\rangle\right]$ & {$\left[X / D_{4}\right]$} & $\coprod_{4}\left[X / \mathbb{Z}_{2}=\langle\bar{b}\rangle\right]$ \\
\hline
\end{tabular}

Table 5. Summary of results for $D_{4} \times\left(\mathbb{Z}_{2}\right)^{2}$ orbifold. In terms of $\mathbb{Z}_{2} \times \mathbb{Z}_{2}=\langle x, y\rangle$, results are symmetric between actions involving $x, y$, and $x y$. In all cases, predictions match partition functions. The reader should note that the orbifold $\left[X / D_{4}\right]$ is reducible, and is a sum of two copies of $\left[X / \mathbb{Z}_{2} \times \mathbb{Z}_{2}\right]$, as indicated in equation (4.160).

Hence, in this case, we predict

$$
\operatorname{QFT}\left([X / \Gamma]_{B, \omega}\right)=\operatorname{QFT}\left(\coprod_{4}\left[X / \mathbb{Z}_{2}=\langle\bar{b}\rangle\right]\right) .
$$

It is straightforward to check this statement at the level of genus-one partition functions. Briefly, for $B(\bar{a})=(+,-,+)$ and $B(\bar{b})$ trivial, with discrete torsion in $D_{4}$ but not $\mathbb{Z}_{2} \times \mathbb{Z}_{2}$, it is straightforward to check that, because of $B(\bar{a})$, all contributions from sectors involving $\bar{a}$ cancel out, and between $z, x, y$, the remaining sectors have multiplicities as, for example

$$
b \square_{1}=\left(4^{3}\right)(\bar{b} \underset{1}{\square})
$$

and the restriction of the $D_{4}$ discrete torsion to $\langle\bar{b}\rangle \subset D_{4}$ is trivial. The genus-one partition function then has the form

$$
\begin{aligned}
Z\left([X / \Gamma]_{B, \omega}\right) & =\frac{4^{3}}{|\Gamma|}\left(1 \square_{1}+\bar{b} \square_{1}+1 \square_{\bar{b}}+\bar{b} \frac{\square}{\bar{b}}\right), \\
& =(2)\left(1 \square_{1}+\bar{b} \square_{1}+1 \square_{\bar{b}}+\bar{b} \square_{\bar{b}}\right), \\
& =(4) Z\left(\left[X / \mathbb{Z}_{2}=\langle\bar{b}\rangle\right]\right)=Z\left(\coprod_{4}\left[X / \mathbb{Z}_{2}=\langle\bar{b}\rangle\right]\right),
\end{aligned}
$$

confirming the prediction.

\subsubsection{Summary}

So far we have worked out predictions for several cases. Computing partition functions is straightforward using the methods already described, so we omit their explicit description; suffice it to say, in all cases, the genus-one partition functions match predictions. We summarize the results in table 5 .

\section{Conclusions}

In this paper, we have described a new set of modular-invariant phase factors for orbifolds with trivially-acting subgroups, generalizing quantum symmetries. We have also described decomposition $[9,16,35,36]$ for orbifolds with quantum symmetries, and detailed both the form of the resulting theories and decomposition in a variety of examples. 
In our next paper [10], we will apply these new phase factors to resolve anomalies in orbifolds.

One matter left for future work is the definition of D-branes and the open string sector in the presence of quantum symmetries. In appendix $\mathrm{C}$ we outline basics, in particular the fact that if $d_{2} B \neq 0$, then associativity of the group action on the D-brane must be (weakly) broken. We intend to explore this in more detail in future work.

Another matter left for future work concerns non-central extensions. In most of this paper, we assume that the full orbifold group $\Gamma$ is a central extension of the effectively-acting group $G$, meaning that the trivially-acting subgroup $K$ lies within the center of $\Gamma$. However, much of the structure we describe also seems to apply to non-central extensions. We outline some basics in appendix D, and intend to explore non-central extensions in more detail in future work.

\section{Acknowledgments}

We would like to thank R. Donagi, T. Pantev, R. Szabo, and Y. Tachikawa for useful conversations. D.R. was partially supported by NSF grant PHY-1820867. E.S. was partially supported by NSF grant PHY-2014086.

\section{A Notes on conventional quantum symmetries in orbifolds}

It is a well-known result that in an orbifold $[X / G]$, there is an abelian $G /[G, G]$ symmetry such that orbifolding by the latter returns $[X /[G, G]]$. Indeed, much of this paper is devoted to generalizing this result. In this appendix we will display this old result in modern language, in the spirit of decomposition [9].

Given some $G$ orbifold of a space $X$, we extend $G$ to $\Gamma=G \times \hat{G}$, where $\hat{G}=G_{\mathrm{ab}}=$ $G /[G, G]$ acts trivially on $X$, and nontrivially on $G$-twisted sectors.

We turn on discrete torsion defined by the two-cocycle $\omega$

$$
\omega\left(\left(g_{1}, g_{1}^{\prime}\right),\left(g_{2}, g_{2}^{\prime}\right)\right)=\alpha\left(g_{1}, g_{2}^{\prime}\right)
$$

where

$$
\alpha: G \times \hat{G} \longrightarrow \mathrm{U}(1)
$$

is a homomorphism in both arguments. (It is straightforward to check that $\omega$ is a two-cocycle, so long as $\alpha$ is a homomorphism in both arguments.) Then the $G$ action on $H^{1}(\hat{G}, \mathrm{U}(1))$ is

$$
(g \cdot \phi)\left(g^{\prime}\right)=\alpha\left(g, g^{\prime}\right) \phi\left(g^{\prime}\right)
$$

We want to show that for some choice of $\alpha$, this action is transitive, meaning that all of $H^{1}(\hat{G}, \mathrm{U}(1))$ is in a single $G$-orbit. Since we can start with the trivial element of $H^{1}\left(G^{\prime}, \mathrm{U}(1)\right)$, namely $\phi\left(g^{\prime}\right)=1$, we just have to show that we can pick $\alpha$ such that every possible $\phi\left(g^{\prime}\right)$ is given by $\alpha\left(g, g^{\prime}\right)$ for some $g$. However, since

$$
H^{1}(\hat{G}, \mathrm{U}(1)) \cong G_{\mathrm{ab}}=G /[G, G]
$$


this is always possible. Let $f: G /[G, G] \rightarrow H^{1}(\hat{G}, \mathrm{U}(1))$ denote the isomorphism, and $p: G \rightarrow G /[G, G]$ the projection, then as $f \circ p$ is surjective, we can take

$$
\alpha\left(g, g^{\prime}\right)=(f \circ p)(g)\left(g^{\prime}\right) .
$$

Now, let us apply the generalized decomposition of [9] to this case. Clearly the restriction of $\omega$ to $K=\hat{G}$ is trivial, but $\beta(\omega) \neq 0$, and in fact is given by [9, equ'n (C.28)]

$$
\begin{aligned}
\beta(\omega)(g, z) & =\frac{\omega\left(g, g^{-1} z\right)}{\omega\left(g^{-1} z, g\right)}=\frac{\omega\left((g, 1),\left(g^{-1}, z\right)\right)}{\omega\left(\left(g^{-1}, z\right),(g, 1)\right)}=\frac{\alpha(g, z)}{\alpha\left(g^{-1}, 1\right)}, \\
& =(f \circ p)(g)(z) .
\end{aligned}
$$

The kernel of this map is $[G, G]$, and there is no cokernel, as it is surjective.

Then, decomposition implies

$$
\operatorname{QFT}\left([X / G \times \hat{G}]_{\omega=\alpha}\right)=\operatorname{QFT}\left(\left[\frac{X \times \operatorname{Coker} \beta(\omega)}{\operatorname{Ker} \beta(\omega)}\right]\right)=\operatorname{QFT}([X /[G, G]) .
$$

\section{B Triviality of $\pi^{*}\left(d_{2} B\right)$ in cohomology}

As observed in section 2.2, not every quantum symmetry $B \in H^{1}\left(G, H^{1}(K, \mathrm{U}(1))\right)$ is determined by discrete torsion. Those which are not determined by discrete torsion have nontrivial images $d_{2} B \in H^{3}(G, \mathrm{U}(1))$. In this appendix we will show that although their images can be nontrivial elements of $H^{3}(G, \mathrm{U}(1))$, their pullbacks $\pi^{*}\left(d_{2} B\right) \in H^{3}(\Gamma, \mathrm{U}(1))$ are trivial as elements of $H^{3}(\Gamma, \mathrm{U}(1))$ - trivial in cohomology, in other words, though not necessarily identically equal to 1 .

Given $B \in H^{1}\left(G, H^{1}(K, \mathrm{U}(1))\right)$ and a section $s: G \rightarrow \Gamma$, define

$$
\lambda\left(g_{1}, g_{2}\right)=B\left(\pi\left(g_{1}\right), s_{1} g_{2} s_{2}^{-1} s_{1}^{-1}\right),
$$

where $g_{i} \in \Gamma$ and $s_{i}=s\left(\pi\left(g_{i}\right)\right)$. Then,

$$
\begin{aligned}
(d \lambda)\left(g_{1}, g_{2}, g_{3}\right)= & \frac{B\left(\pi\left(g_{2}\right), s_{2} g_{3} s_{3}^{-1} s_{2}^{-1}\right) B\left(\pi\left(g_{1}\right), s_{1} g_{2} g_{3} s_{23}^{-1} s_{1}^{-1}\right)}{B\left(\pi\left(g_{1} g_{2}\right), s_{12} g_{3} s_{3}^{-1} s_{12}^{-1}\right) B\left(\pi\left(g_{1}\right), s_{1} g_{2} s_{2}^{-1} s_{1}^{-1}\right.}= \\
= & B\left(\pi\left(g_{1}\right), s_{1} g_{2} g_{3} s_{23}^{-1} s_{1}^{-1} \cdot s_{12} s_{3} g_{3}^{-1} s_{12}^{-1} \cdot s_{1} s_{2} g_{2}^{-1} s_{1}^{-1}\right) \\
& \cdot B\left(\pi\left(g_{2}\right), s_{2} g_{3} s_{3}^{-1} s_{2}^{-1} \cdot s_{1}^{-1} s_{12} s_{3} g_{3}^{-1} s_{12}^{-1} s_{1}\right), \\
= & B\left(\pi\left(g_{1}\right), s_{1} s_{2} g_{2}^{-1} s_{1}^{-1} \cdot s_{1} g_{2} g_{3} s_{23}^{-1} s_{1}^{-1} \cdot s_{12} s_{3} g_{3}^{-1} s_{12}^{-1}\right) \\
& \cdot B\left(\pi\left(g_{2}\right), s_{2} g_{3} s_{3}^{-1} s_{2}^{-1} \cdot s_{1}^{-1} s_{12} s_{2}^{-1} \cdot s_{2} s_{3} g_{3}^{-1} s_{2}^{-1} \cdot s_{2} s_{12}^{-1} s_{1}\right), \\
= & B\left(\pi\left(g_{1}\right), s_{1} s_{2} s_{12}^{-1} \cdot s_{12} g_{3} s_{23}^{-1} s_{1}^{-1} \cdot s_{12} s_{2}^{-1} s_{1}^{-1} \cdot s_{1} s_{2} s_{3} g_{3}^{-1} s_{12}^{-1}\right), \\
= & B\left(\pi\left(g_{1}\right), s_{1} s_{2} s_{3} s_{23}^{-1} s_{1}^{-1}\right), \\
= & \left(d_{2} B\right)\left(\pi\left(g_{1}\right), \pi\left(g_{2}\right), \pi\left(g_{3}\right)\right)=\left(\pi^{*} d_{2} B\right)\left(g_{1}, g_{2}, g_{3}\right) .
\end{aligned}
$$

In the expression above, we have not assumed that $\Gamma$ is a central extension, instead using the more general conventions outlined in appendix D.

In any event, we now see that $\pi^{*}\left(d_{2} B\right)$ is trivial in cohomology, even when $\Gamma$ is not a central extension of $G$. 


\section{Open string sector}

In this appendix we outline some ideas regarding open string sectors in these theories with quantum symmetries. Briefly, ordinarily one would describe D-branes in terms of (possibly projective) equivariant structures on some sheaf or bundle on the covering space; however, if $d_{2} B \neq 1$, then associativity is broken. That said, associativity is broken by $\pi^{*}\left(d_{2} B\right)$, which as we saw in appendix B is trivial in cohomology, so associativity holds 'up to homotopy,' and is only broken in a weak sense.

In this appendix, we will only outline such weakly associative versions of (projectively) equivariant structures. We leave a more detailed examination of the open string sector for future work.

To define D-branes, let us attempt to proceed in the same fashion as for discrete torsion in [50-52], in which the action of the group $\Gamma$ on the Chan-Paton factors is twisted by a group cochain.

Let $\omega$ be a 2 -cochain in $\Gamma$ such that

$$
(d \omega)\left(g_{1}, g_{2}, g_{3}\right)=B\left(\pi\left(g_{1}\right), s\left(\pi\left(g_{2}\right)\right) s\left(\pi\left(g_{3}\right)\right) s\left(\pi\left(g_{2} g_{3}\right)\right)^{-1}=\pi^{*}\left(d_{2} B\right)\left(g_{1}, g_{2}, g_{3}\right)^{-1},\right.
$$

where $s$ denotes a normalized section $s: G \rightarrow \Gamma$ (normalized meaning that $s(1)=1$ ), and where we also assume that the cochain is normalized so that

$$
\omega(g, 1)=\omega(1, g)=1 .
$$

(Note in passing that this includes as a special case $\omega$ that are coclosed, corresponding to elements of discrete torsion.) Such a cochain $\omega$ exists precisely because $\pi^{*}\left(d_{2} B\right)$ is trivial in cohomology, even if $d_{2} B$ itself is not.

Let $\phi$ be a twisted representation of $\Gamma$, twisted in the sense that

$$
\phi\left(g_{1}\right) \phi\left(g_{2}\right)=\omega\left(g_{1}, g_{2}\right) \phi\left(g_{1} g_{2}\right) .
$$

(The normalization condition allows us to consistently identify $\phi(1)$ with the identity.) This is very similar to a projective representation, except that $\omega$ is a cochain rather than a cocycle. As a result, multiplication is not quite associative:

$$
\begin{aligned}
& \phi\left(g_{1}\right)\left(\phi\left(g_{2}\right) \phi\left(g_{3}\right)\right)=\omega\left(g_{1}, g_{2} g_{3}\right) \omega\left(g_{2}, g_{3}\right) \phi\left(g_{1} g_{2} g_{3}\right), \\
& \left(\phi\left(g_{1}\right) \phi\left(g_{2}\right)\right) \phi\left(g_{3}\right)=\omega\left(g_{1}, g_{2}\right) \omega\left(g_{1} g_{2}, g_{3}\right) \phi\left(g_{1} g_{2} g_{3}\right),
\end{aligned}
$$

hence

$$
\phi\left(g_{1}\right)\left(\phi\left(g_{2}\right) \phi\left(g_{3}\right)\right)=(d \omega)\left(g_{1}, g_{2}, g_{3}\right)\left(\phi\left(g_{1}\right) \phi\left(g_{2}\right)\right) \phi\left(g_{3}\right) .
$$

If $d \omega=1$, as happens in the case of discrete torsion, then we see that multiplication of the $\phi$ is associative, and we see that the $\phi$ form a projective representation of $\Gamma$.

However, if $d \omega \neq 1$, then associativity does not hold, and we do not have a projective representation of $\Gamma$. Furthermore, if we implement this group action in boundary OPEs between topological defect lines at the boundary, this means that such boundary OPEs are nonassociative.

This may seem rather strange, but nonassociative boundary OPEs have been considered previously in [53-56] in the context of D-branes in the presence of ${ }^{5}$ nontrivial $H$ flux. In

\footnotetext{
${ }^{5}$ We would like to thank R. Szabo for a useful discussion of this matter.
} 
fact, the analogy can be made more precise. Discrete torsion in orbifolds can be described in terms of $B$ fields, see [51, 52], and the image of $H^{1}\left(G, H^{1}(K, \mathrm{U}(1))\right)$ in $H^{3}(G, \mathrm{U}(1))$ is an analogue of the curvature $H$. If the element of $H^{1}\left(G, H^{1}(K, \mathrm{U}(1))\right)$ is in the image of discrete torsion, then we get an ordinary associative projective equivariant structure. If the element of $H^{1}\left(G, H^{1}(K, \mathrm{U}(1))\right)$ has a nonzero image in $H^{3}(G, \mathrm{U}(1))$, which morally is the analogue of a non-trivial $H$, then the equivariant structure is nonassociative, in precise analogy with the nonassociative boundary OPEs arising in cases with nontrivial $H$ flux in $[53-56]$.

Now, to be clear, no examples exist in which $\phi$ acts as a linear transformation on a finite-dimensional vector space. In such a case, $\phi(k)$ is simply a finite-dimensional matrix, and matrix multiplication is always associative in finite dimensions. However, if the vector space is infinite-dimensional, then, matrix multiplication can be nonassociative, see for example [57-61]. An example from [57] is defined by the matrices

$$
V=\left[\begin{array}{cccc}
1 & 1 & 1 & \cdots \\
0 & 1 & 1 & \cdots \\
0 & 0 & 1 & \cdots \\
\vdots & \vdots & \vdots & \ddots
\end{array}\right], \quad U=\left[\begin{array}{rrrrr}
1 & -1 & 0 & 0 & \cdots \\
0 & 1 & -1 & 0 & \cdots \\
0 & 0 & 1 & -1 & \cdots \\
\vdots & \vdots & \vdots & \vdots & \ddots
\end{array}\right], \quad A=\left[\begin{array}{rrrr}
0 & 0 & 0 & \cdots \\
-1 & 0 & 0 & \cdots \\
-1 & -1 & 0 & \cdots \\
\vdots & \vdots & \vdots & \ddots
\end{array}\right]
$$

where in $U$ each row has one successive $(+1,-1)$ pair with other entries equal to zero. It is easy to see that $U A$ and $V U$ are both the identity, hence

$$
V(U A)=V \neq A=(V U) A
$$

In passing, physically this would require infinite-rank Chan-Paton factors in the case that the quantum symmetry does not arise from discrete torsion. Similarly, in the case that the curvature $H$ of the $B$ field is nonzero in de Rham cohomology, any sheaf twisted by $H$ also has infinite rank (see e.g. [62, section 4.1]), in line with the role of $H$ in nonassociative open string products in [53-56, 63-65].

In principle, given such a nonassociative equivariant structure, one could then construct closed-string phases in much the same fashion described for discrete torsion in e.g. [66], though we shall not try to do so here.

We have outlined one possible way to understand open string sectors in orbifolds with $B$ such that $d_{2} B \neq 1$, breaking associativity, but there are other approaches. For example,

- Another approach is described in [67, section 2.3], where groups $G$ with an anomaly in $H^{3}(G, \mathrm{U}(1))$ are described as groupoids, which have representations on 2-vector spaces. Similar approaches are described in [68, 69], [70, sections 8-9], [71, appendix E].

- Non-associative equivariant structures in a different context are described in [72-75].

We leave a detailed examination of open string sectors for future work. 


\section{Non-central extensions}

In the rest of this paper, we have focused on central extensions. However, some preliminary computations suggest that similar results should hold for non-central extensions. We do not claim a complete understanding of such cases, but collect here a few pertinent results.

\section{D.1 Basics}

Consider an orbifold of a space $X$ by a group $\Gamma$, where a subgroup $K \subset \Gamma$ acts trivially, with effectively-acting coset $G=\Gamma / K$ :

$$
1 \longrightarrow K \longrightarrow \Gamma \stackrel{\pi}{\longrightarrow} G \longrightarrow 1 \text {. }
$$

We do not assume that $K$ is necessarily central.

We can describe the quantum symmetry as follows. For $g \in \Gamma, k \in K$, the quantum symmetry is a phase assigned to twist fields, depending on their projection to $G$, which we take to obey

$$
B\left(\pi\left(g_{1}\right) \pi\left(g_{2}\right), k\right)=B\left(\pi\left(g_{1}\right), k\right) B\left(\pi\left(g_{2}\right), g_{1}^{-1} k g_{1}\right) .
$$

As the action of the identity should be trivial, we also require

$$
B(1, k)=1=B(\bar{g}, 1)
$$

for $\bar{g} \in G, k \in K$. For $g, h \in \Gamma, z \in K$, and $s: G \rightarrow \Gamma$ a section (meaning $\pi \circ s$ is the identity on $G$ ), the quantum symmetry is a relation

$$
\begin{gathered}
g z \square_{h}=B\left(\pi(h), s(\pi(h)) z s(\pi(h))^{-1}\right)(g \underset{h}{\square}), \\
g \square_{h z}=B\left(\pi(g), s(\pi(g)) z s(\pi(g))^{-1}\right)^{-1}(g \underset{h}{\square}),
\end{gathered}
$$

where $B: G \times K \rightarrow \mathrm{U}(1)$ is a map such that

$$
\begin{aligned}
& B\left(\bar{g}_{1} \bar{g}_{2}, k\right)=B\left(\bar{g}_{1}, k\right) B\left(\bar{g}_{2}, s\left(\bar{g}_{1}\right)^{-1} k s\left(\bar{g}_{1}\right)\right), \\
& B\left(\bar{g}, k_{1} k_{2}\right)=B\left(\bar{g}, k_{1}\right) B\left(\bar{g}, k_{2}\right),
\end{aligned}
$$

for $\bar{g}, \bar{g}_{1,2} \in G$.

The phase above is independent of the choice of section $s$ : over any $\bar{h} \in G$, the value of any two choices of section will differ by an element $k \in K$, and note

$$
\begin{aligned}
B\left(\pi(h),(s k) z(s k)^{-1}\right) & =B\left(\pi(h),\left(s k s^{-1}\right)\left(s z s^{-1}\right)\left(s k^{-1} s^{-1}\right)\right), \\
& =B\left(\pi(h), s k s^{-1}\right) B\left(\pi(h), s k^{-1} s^{-1}\right) B\left(\pi(h), s z s^{-1}\right), \\
& =B\left(\pi(h), s z s^{-1}\right) .
\end{aligned}
$$

Given a section, we can interpret these quantum symmetries as defining discrete-torsionlike phases associated to sectors in the effective $G$, specifically,

$$
g \square_{h}=\epsilon(g, h)(\pi(g) \underset{\pi(h)}{\square}),
$$


for commuting $g, h \in \Gamma$, where

$$
\epsilon(g, h)=\frac{B\left(\pi(h), s_{h} s_{g}^{-1} g s_{h}^{-1}\right)}{B\left(\pi(g), s_{g} s_{h}^{-1} h s_{g}^{-1}\right),}
$$

where we have abbreviated $s_{g}=s(\pi(g))$. As a consistency check, note that

$$
\begin{aligned}
\frac{g z \underline{h}}{g \square} & =\frac{\epsilon(g z, h)}{\epsilon(g, h)}, \\
& =\frac{B\left(\pi(h), s_{h} s_{g}^{-1} g z s_{h}^{-1}\right)}{B\left(\pi(h), s_{h} s_{g}^{-1} g s_{h}^{-1}\right)} \\
& =B\left(\pi(h), s_{h} s_{g}^{-1} g z\left(s_{g}^{-1} g\right)^{-1} s_{h}^{-1}\right), \\
& =B\left(\pi(h), s_{h} s_{g}^{-1} g s_{h}^{-1}\right) B\left(\pi(h), s_{h} z s_{h}^{-1}\right) B\left(\pi(h), s_{h} g^{-1} s_{g} s_{h}^{-1}\right), \\
& =B\left(\pi(h), s_{h} z s_{h}^{-1}\right),
\end{aligned}
$$

matching the original definition (D.4).

Now, let us consider the genus-one phases $\epsilon(g, h)$ arising from a quantum symmetry in a $\Gamma$-orbifold, given in equation (D.11). It is straightforward to show that $\epsilon(g, g)=1$ and $\epsilon(g, h)=\epsilon(h, g)^{-1}$. It is also straightforward to see that

$$
\begin{aligned}
\epsilon\left(g, h_{1} h_{2}\right)=\epsilon\left(g, h_{1}\right) & \epsilon\left(g, h_{2}\right) B\left(\pi(g), s_{g} s_{1}^{-1} h_{1} s_{2}^{-1} h_{1}^{-1} s_{12} s_{g}^{-1}\right) \\
\cdot & B\left(\pi\left(h_{1}\right), s_{12} s_{g}^{-1} g s_{12}^{-1} s_{1} g^{-1} s_{g} s_{1}^{-1}\right) \\
\cdot & B\left(\pi\left(h_{2}\right), s_{1}^{-1} s_{12} s_{g}^{-1} g s_{12}^{-1} s_{1} s_{2} g^{-1} s_{g} s_{2}^{-1}\right) .
\end{aligned}
$$

Using the fact that

$$
\begin{aligned}
& s_{1}^{-1} s_{12} s_{g}^{-1} g s_{12}^{-1} s_{1} s_{2} g^{-1} s_{g} s_{2}^{-1} \\
& =\left(s_{1}^{-1} s_{12} s_{2}^{-1}\right)\left(s_{2} s_{g}^{-1} g s_{2}^{-1}\right)\left(s_{2} s_{12}^{-1} s_{1}\right)\left(s_{2} g^{-1} s_{g} s_{2}^{-1}\right),
\end{aligned}
$$

where each factor on the right lies in $K$, we can then write

$$
\begin{aligned}
& B\left(\pi\left(h_{2}\right), s_{1}^{-1} s_{12} s_{g}^{-1} g s_{12}^{-1} s_{1} s_{2} g^{-1} s_{g} s_{2}^{-1}\right) \\
& =B\left(\pi\left(h_{2}\right), s_{1}^{-1} s_{12} s_{2}^{-1}\right) B\left(\pi\left(h_{2}\right), s_{2} s_{12}^{-1} s_{1}\right) B\left(\pi\left(h_{2}\right), s_{2} s_{g}^{-1} g s_{2}^{-1}\right) B\left(\pi\left(h_{2}\right), s_{2} g^{-1} s_{g} s_{2}^{-1}\right), \\
& =1 .
\end{aligned}
$$

Thus, we can write

$$
\begin{aligned}
\epsilon\left(g, h_{1} h_{2}\right)=\epsilon\left(g, h_{1}\right) & \epsilon\left(g, h_{2}\right)\left(d_{2} B\right)\left(\pi(g), \pi\left(h_{1}\right) \pi\left(h_{2}\right)\right)^{-1} \\
\cdot & B\left(\pi(g),\left(s_{g} s_{1}^{-1} h_{1} s_{2}^{-1} h_{1}^{-1} s_{12}\right)\left(s_{12}^{-1} s_{2} s_{1} s_{g}^{-1}\right)\right) \\
\cdot & B\left(\pi\left(h_{1}\right), s_{12} s_{g}^{-1} g s_{12}^{-1} s_{1} g^{-1} s_{g} s_{1}^{-1}\right),
\end{aligned}
$$


where $d_{2}$ is the map $H^{1}\left(G, H^{1}(K, \mathrm{U}(1))\right) \rightarrow H^{3}(G, \mathrm{U}(1))$ appearing in section 2.2 , so that explicitly

$$
\left(d_{2} B\right)\left(\pi(g), \pi\left(h_{1}\right), \pi\left(h_{2}\right)\right)=B\left(\pi(g), s_{g} s_{1}^{-1} s_{2}^{-2} s_{12} s_{g}^{-1}\right) .
$$

Now, to settle all issues associated with quantum symmetries in non-central extensions, there are several more things we need to do at this point. For example, we need to demonstrate that $B$ only depends upon the conjugacy class of $\bar{g}$, to show that $B$ acts on twist fields of $G$; we need to elaborate on what circumstances this is a symmetry, when the correlation functions are invariant; and we need to discuss why physically in this more general case the quantum symmetries are classified by $H^{1}\left(G, H^{1}(K, \mathrm{U}(1))\right.$, defined with the crossed module condition. We leave all of that for future work, and focus here on merely outlining a few other matters. We conclude this appendix with an example.

\section{D.2 Example}

Consider the case that $K=\mathbb{Z}_{4}=\langle i\rangle \subset \Gamma=\mathbb{H}$, the group of quaternions. We have the short exact sequence

$$
1 \longrightarrow \mathbb{Z}_{4} \longrightarrow \mathbb{H} \longrightarrow \mathbb{Z}_{2} \longrightarrow 1
$$

Specifically, consider the orbifold $[X / \Gamma]_{B}$, where the quantum symmetry $B$ is defined to be the nontrivial element of $H^{1}\left(G, H^{1}(K, \mathrm{U}(1))\right)$.

Explicitly, if we let $\xi$ denote the generator of $G=\mathbb{Z}_{2}$, and $i$ the generator of $K=\mathbb{Z}_{4}$, then we take

$$
B(\xi, k)=\exp (\pi i / 2) .
$$

Let us quickly check that this has the crossed homomorphism structure. Take the section $s: G \rightarrow \Gamma$ to be defined by $s(\xi)=j$, so that $s(\xi)^{-1}=-j$. Then,

$$
\begin{aligned}
B(\xi, i) & =\exp (\pi i / 2), \\
B\left(\xi, s(\xi)^{-1}(i) s(\xi)\right) & =B(\xi,(-j)(i)(j))=B(\xi,-j k)=B\left(\xi, i^{3}\right), \\
& =\exp (3 \pi i / 2) \\
B\left(\xi^{2}, i\right) & =1
\end{aligned}
$$

and indeed one can see that

$$
B\left(\xi^{2}, i\right)=B(\xi, i) B\left(\xi, s(\xi)^{-1}(i) s(\xi)\right)
$$

as expected.

Using this quantum symmetry, one can compute

$$
\begin{aligned}
(-) \square_{ \pm j, \pm k} & =B\left(\xi, i^{2}\right)\left(1_{ \pm j, \pm k}^{\square}\right)=(-)\left(1_{ \pm j, \pm k}^{\square_{j}}\right), \\
-j \square_{ \pm j} & =B\left(\xi, i^{2}\right)(j \underset{ \pm j}{\square})=(-)(j \square), \\
-k \square_{ \pm j} & =(-)(k \underset{ \pm k}{\square}),
\end{aligned}
$$


hence the genus-one partition function is given by

$$
\begin{aligned}
Z\left([X / \mathbb{H}]_{B}\right) & =\frac{1}{|\mathbb{H}|} \sum_{g h=h g} g \square_{h} \\
& =\frac{4^{2}}{8}(1 \underset{1}{\square})=2(1 \underset{\square}{1})
\end{aligned}
$$

since all genus-one sectors that do not project to the trivial sector cancel out. Thus, at the level of genus-one partition functions, we find

$$
[X / \mathbb{H}]_{B}=\coprod_{2} X=X \coprod X
$$

Open Access. This article is distributed under the terms of the Creative Commons Attribution License (CC-BY 4.0), which permits any use, distribution and reproduction in any medium, provided the original author(s) and source are credited.

\section{References}

[1] D.G. Robbins, E. Sharpe and T. Vandermeulen, Anomalies, extensions, and orbifolds, Phys. Rev. D 104 (2021) 085009 [arXiv:2106. 00693] [INSPIRE].

[2] J. Wang, X.-G. Wen and E. Witten, Symmetric Gapped Interfaces of SPT and SET States: Systematic Constructions, Phys. Rev. X 8 (2018) 031048 [arXiv: 1705. 06728] [INSPIRE].

[3] L. Bhardwaj and Y. Tachikawa, On finite symmetries and their gauging in two dimensions, JHEP 03 (2018) 189 [arXiv: 1704.02330] [INSPIRE].

[4] Y. Tachikawa, On gauging finite subgroups, SciPost Phys. 8 (2020) 015 [arXiv:1712.09542] [INSPIRE].

[5] C.-M. Chang, Y.-H. Lin, S.-H. Shao, Y. Wang and X. Yin, Topological Defect Lines and Renormalization Group Flows in Two Dimensions, JHEP 01 (2019) 026 [arXiv:1802.04445] [INSPIRE].

[6] D. Robbins and T. Vandermeulen, Orbifolds from Modular Orbits, Phys. Rev. D 101 (2020) 106021 [arXiv: 1911.05172] [INSPIRE].

[7] D. Robbins and T. Vandermeulen, Modular Orbits at Higher Genus, JHEP 02 (2020) 113 [arXiv: 1911.06306] [INSPIRE].

[8] Y. Tachikawa, TASI 2019 lectures, available at https://member.ipmu.jp/yuji.tachikawa/lectures/2019-top-anom/tasi2019.pdf.

[9] D. Robbins, E. Sharpe and T. Vandermeulen, A generalization of decomposition in orbifolds, JHEP 10 (2021) 134 [arXiv:2101.11619] [InSPIRE].

[10] D. Robbins, E. Sharpe and T. Vandermeulen, Anomaly resolution via decomposition, to appear.

[11] P.H. Ginsparg, Applied conformal field theory, in Les Houches Summer School in Theoretical Physics: Fields, Strings, Critical Phenomena, (1988) [hep-th/9108028] [INSPIRE].

[12] C. Vafa, Quantum Symmetries of String Vacua, Mod. Phys. Lett. A 4 (1989) 1615 [INSPIRE].

[13] T. Pantev and E. Sharpe, Notes on gauging noneffective group actions, hep-th/0502027 [INSPIRE]. 
[14] T. Pantev and E. Sharpe, String compactifications on Calabi-Yau stacks, Nucl. Phys. B 733 (2006) 233 [hep-th/0502044] [INSPIRE].

[15] T. Pantev and E. Sharpe, GLSM's for Gerbes (and other toric stacks), Adv. Theor. Math. Phys. 10 (2006) 77 [hep-th/0502053] [InSPIRE].

[16] S. Hellerman, A. Henriques, T. Pantev, E. Sharpe and M. Ando, Cluster decomposition, T-duality, and gerby CFT's, Adv. Theor. Math. Phys. 11 (2007) 751 [hep-th/0606034] [INSPIRE].

[17] E. Andreini, Y. Jiang, H.-H. Tseng, On Gromov-Witten theory of root gerbes, arXiv:0812.4477.

[18] E. Andreini, Y. Jiang and H.-H. Tseng, Gromov-Witten theory of product stacks, Commun. Anal. Geom. 24 (2016) 223 [arXiv:0905.2258].

[19] E. Andreini, Y. Jiang and H.-H. Tseng, Gromov-Witten theory of root gerbes I: structure of genus 0 moduli spaces, J. Diff. Geom. 99 (2015) 1 [arXiv:0907.2087].

[20] H.-H. Tseng, On degree zero elliptic orbifold Gromov-Witten invariants, Int. Math. Res. Not. 2011 (2011) 2444 [arXiv:0912.3580].

[21] A. Gholampour and H.-H. Tseng, On Donaldson-Thomas invariants of threefold stacks and gerbes, Proc. Am. Math. Soc. 141 (2013) 191 [arXiv:1001.0435].

[22] X. Tang and H.-H. Tseng, Duality theorems of etale gerbes on orbifolds, Adv. Math. 250 (2014) 496 [arXiv: 1004.1376] [INSPIRE].

[23] A. Caldararu, J. Distler, S. Hellerman, T. Pantev and E. Sharpe, Non-birational twisted derived equivalences in abelian GLSMs, Commun. Math. Phys. 294 (2010) 605 [arXiv:0709.3855] [INSPIRE].

[24] K. Hori, Duality In Two-Dimensional $(2,2)$ Supersymmetric Non-Abelian Gauge Theories, JHEP 10 (2013) 121 [arXiv:1104.2853] [INSPIRE].

[25] N.M. Addington, E.P. Segal and E. Sharpe, D-brane probes, branched double covers, and noncommutative resolutions, Adv. Theor. Math. Phys. 18 (2014) 1369 [arXiv:1211.2446] [INSPIRE].

[26] E. Sharpe, Predictions for Gromov-Witten invariants of noncommutative resolutions, J. Geom. Phys. 74 (2013) 256 [arXiv:1212.5322] [INSPIRE].

[27] K. Hori and J. Knapp, Linear sigma models with strongly coupled phases - one parameter models, JHEP 11 (2013) 070 [arXiv: 1308.6265] [INSPIRE].

[28] K. Hori and J. Knapp, A pair of Calabi-Yau manifolds from a two parameter non-Abelian gauged linear sigma model, arXiv:1612.06214 [INSPIRE].

[29] J. Knapp and E. Sharpe, GLSMs, joins, and nonperturbatively-realized geometries, JHEP 12 (2019) 096 [arXiv: 1907.04350] [InSPIRE].

[30] W. Gu and E. Sharpe, A proposal for nonabelian mirrors, arXiv:1806.04678 [INSPIRE].

[31] Z. Chen, W. Gu, H. Parsian and E. Sharpe, Two-dimensional supersymmetric gauge theories with exceptional gauge groups, Adv. Theor. Math. Phys. 24 (2020) 67 [arXiv:1808.04070] [INSPIRE].

[32] W. Gu, H. Parsian and E. Sharpe, More non-Abelian mirrors and some two-dimensional dualities, Int. J. Mod. Phys. A 34 (2019) 1950181 [arXiv:1907.06647] [InSPIRE].

[33] W. Gu, E. Sharpe and H. Zou, Notes on two-dimensional pure supersymmetric gauge theories, JHEP 04 (2021) 261 [arXiv: 2005.10845] [INSPIRE]. 
[34] L.B. Anderson, B. Jia, R. Manion, B. Ovrut and E. Sharpe, General aspects of heterotic string compactifications on stacks and gerbes, Adv. Theor. Math. Phys. 19 (2015) 531 [arXiv: 1307.2269] [INSPIRE].

[35] E. Sharpe, Undoing decomposition, Int. J. Mod. Phys. A 34 (2020) 1950233 [arXiv: 1911.05080] [INSPIRE].

[36] Y. Tanizaki and M. Ünsal, Modified instanton sum in QCD and higher-groups, JHEP 03 (2020) 123 [arXiv: 1912.01033] [INSPIRE].

[37] A. Cherman and T. Jacobson, Lifetimes of near eternal false vacua, Phys. Rev. D 103 (2021) 105012 [arXiv: 2012.10555] [INSPIRE].

[38] R. Eager and E. Sharpe, Elliptic Genera of Pure Gauge Theories in Two Dimensions with Semisimple Non-Simply-Connected Gauge Groups, Commun. Math. Phys. 387 (2021) 267 [arXiv: 2009.03907] [INSPIRE].

[39] Z. Komargodski, K. Ohmori, K. Roumpedakis and S. Seifnashri, Symmetries and strings of adjoint $Q C D_{2}$, JHEP 03 (2021) 103 [arXiv:2008.07567] [INSPIRE].

[40] D. Gaiotto and J. Kulp, Orbifold groupoids, JHEP 02 (2021) 132 [arXiv:2008.05960] [INSPIRE].

[41] G. Hochschild, Basic constructions in group extension theory, in Contributions to algebra: a collection of papers dedicated to Ellis Kolchin, H. Bass, P.J. Cassidy and J. Kovacic eds., Academic Press, New York (1977), pp. 183-201.

[42] G. Hochschild and J.-P. Serre, Cohomology of group extensions, Trans. Am. Math. Soc. 74 (1953) 110.

[43] C. Weibel, An introduction to homological algebra, Cambridge University Press, Cambridge, U.K. (1994) [DOI].

[44] J. Neukirch, A. Schmidt and K. Wingberg, Cohomology of number fields, second edition, Springer-Verlag, Berlin (2008) [DOI].

[45] P. Gille and T. Szamuely, Central simple algebras and Galois cohomology, Cambridge Studies in Advanced Mathematics, vol. 101, Cambridge University Press, Cambridge (2006) [DOI].

[46] C. Vafa, Modular Invariance and Discrete Torsion on Orbifolds, Nucl. Phys. B 273 (1986) 592 [INSPIRE].

[47] P. Etingof, S. Gelaki, D. Nikshych and V. Ostrik, Tensor categories, Mathematical Surveys and Monographs, vol. 205, American Mathematical Society, Providence, Rhode Island (2015).

[48] L. Müller, Extended Functorial Field Theories and Anomalies in Quantum Field Theories, arXiv:2003.08217 [INSPIRE].

[49] E. Sharpe, Discrete torsion and shift orbifolds, Nucl. Phys. B 664 (2003) 21 [hep-th/0302152] [INSPIRE].

[50] M.R. Douglas, D-branes and discrete torsion, hep-th/9807235 [INSPIRE].

[51] E.R. Sharpe, Discrete torsion, Phys. Rev. D 68 (2003) 126003 [hep-th/0008154] [INSPIRE].

[52] E.R. Sharpe, Recent developments in discrete torsion, Phys. Lett. B 498 (2001) 104 [hep-th/0008191] [INSPIRE].

[53] L. Cornalba and R. Schiappa, Nonassociative star product deformations for D-brane world volumes in curved backgrounds, Commun. Math. Phys. 225 (2002) 33 [hep-th/0101219] [INSPIRE]. 
[54] M. Herbst, A. Kling and M. Kreuzer, Star products from open strings in curved backgrounds, JHEP 09 (2001) 014 [hep-th/0106159] [INSPIRE].

[55] M. Herbst, A. Kling and M. Kreuzer, Noncommutative tachyon action and D-brane geometry, JHEP 08 (2002) 010 [hep-th/0203077] [INSPIRE].

[56] M. Herbst, A. Kling and M. Kreuzer, Cyclicity of nonassociative products on D-branes, JHEP 03 (2004) 003 [hep-th/0312043] [inSPIRE].

[57] D. Bossaller and S.R. López-Permouth, On the associativity of infinite matrix multiplication, arXiv: 1803.09779.

[58] M. Bernkopf, A history of infinite matrices, Arch. Hist. Exact Sci. 4 (1968) 308.

[59] K. Keremedis and A. Abian, On the associativity and commutativity of multiplication of infinite matrices, Int. J. Math. Educ. Sci. Technol. 19 (1988) 175.

[60] L.M. Al-Essa, S.R. López-Permouth and N.M. Muthana, Modules over infinite-dimensional algebras, Linear Multilinear Alg. 66 (2018) 488.

[61] R. Cooke, Infinite matrices and sequence spaces, Dover, New York (1955).

[62] J.-L. Brylinski, Loop spaces, characteristic classes, and geometric quantization, Birkhäuser, Boston (1993) [DOI].

[63] R. Blumenhagen, M. Fuchs, F. Haßler, D. Lüst and R. Sun, Non-associative Deformations of Geometry in Double Field Theory, JHEP 04 (2014) 141 [arXiv:1312.0719] [InSPIRE].

[64] R. Jackiw, Three-cocycle in mathematics and physics, Phys. Rev. Lett. 54 (1985) 159 [INSPIRE].

[65] M. Gunaydin and B. Zumino, Magnetic charge and non-associative algebras, in Old and new problems in fundamental physics: meeting in honour of G.C. Wick, Quaderni, Pisa, Scuola Normale Superiore (1986), pp. 43-53 [INSPIRE].

[66] P.S. Aspinwall, A Note on the equivalence of Vafa's and Douglas's picture of discrete torsion, JHEP 12 (2000) 029 [hep-th/0009045] [INSPIRE].

[67] S. Willerton, The twisted Drinfeld double of a finite group via gerbes and finite groupoids, Algebr. Geom. Topol. 8 (2008) 1419 [math/0503266].

[68] P. Roche, V. Pasquier and R. Dijkgraaf, QuasiHopf algebras, group cohomology and orbifold models, Nucl. Phys. B Proc. Suppl. 18 (1990) 60 [InSPIRE].

[69] J.C. Baez, A. Baratin, L. Freidel and D.K. Wise, Infinite-Dimensional Representations of 2-Groups, Mem. Am. Math. Soc. 1032 (2012) 1 [arXiv:0812.4969] [INSPIRE].

[70] D.S. Freed, Higher algebraic structures and quantization, Commun. Math. Phys. 159 (1994) 343 [hep-th/9212115] [inSPIRE].

[71] G.W. Moore and N. Seiberg, Classical and Quantum Conformal Field Theory, Commun. Math. Phys. 123 (1989) 177 [InSPIRE].

[72] S. Bunk, L. Müller and R.J. Szabo, Geometry and 2-Hilbert Space for Nonassociative Magnetic Translations, Lett. Math. Phys. 109 (2019) 1827 [arXiv:1804.08953] [inSPIRE].

[73] S. Bunk, L. Müller and R.J. Szabo, Smooth 2-Group Extensions and Symmetries of Bundle Gerbes, Commun. Math. Phys. 384 (2021) 1829 [arXiv:2004.13395] [INSPIRE].

[74] J. Mickelsson, Non-associative magnetic translations: A QFT construction, arXiv: 1905.01944 [INSPIRE].

[75] J. Mickelsson and M. Murray, Non associative magnetic translations from parallel transport in projective Hilbert bundles, J. Geom. Phys. 163 (2021) 104152 [arXiv:2011.11431] [INSPIRE]. 